\title{
True Color Imagery Rendering for Himawari-8 with a Color Reproduction Approach Based on the CIE XYZ Color System
}

\author{
Hidehiko MURATA, Kotaro SAITOH, and Yasuhiko SUMIDA \\ Meteorological Satellite Center, Japan Meteorological Agency, Tokyo, Japan
}

(Manuscript received 5 June 2017, in final form 20 April 2018)

\begin{abstract}
The combination of three visible bands of the Advanced Himawari Imager (AHI) aboard Japan Meteorological Agency's (JMA) new-generation Himawari-8 and Himawari-9 geostationary meteorological satellites enables the production of true color imagery. True color is intuitively understandable to human analysts and beneficial for monitoring surface and atmospheric features. It is particularly useful when applied to frequent observations from a geostationary platform. In this article, we report on an application of a color reproduction approach based on the International Commission on Illumination (CIE) 1931 XYZ color system to imagery rendering. This approach allows the consideration of primary color (RGB) differences among satellite and output devices, which in turn cause differences in the colors reproduced. The RGB signals observed by the AHI are converted to XYZ tristimulus values, which are independent of the devices themselves, and then reconverted to RGB signals for output devices via the application of $3 \times 3$ conversion matrices. This article also covers an objective technique for the evaluation of the accuracy of XYZ values. The evaluation indicated that the combination of AHI native RGB bands is suboptimal for obtaining XYZ values as is, whereas a combination in which the green band is replaced by a pseudo band with a central wavelength of around $0.555 \mu \mathrm{m}$ is optimal. The pseudo band is generated via regression with existing visible and near-infrared bands as predictor variables. The imagery produced using this approach was termed True Color Reproduction (TCR). This approach is applicable to other satellites that have several bands in the visible to near-infrared spectral range, and it has the potential for development toward the production of standardized sensor-independent true color imagery.
\end{abstract}

Keywords Himawari-8; Advanced Himawari Imager; true color; color reproduction; CIE XYZ; chromaticity

\section{Introduction}

The Japan Meteorological Agency's (JMA) newgeneration Himawari-8 and Himawari-9 geostationary meteorological satellites (Bessho et al. 2016) feature the Advanced Himawari Imager (AHI) with 16 spectral bands (three visible, three near-infrared, and ten infrared; Table 1). The specific combination of the visible bands B03 (red: $0.64 \mu \mathrm{m}$ ), B02 (green: 0.51

Corresponding author: Hidehiko Murata, Meteorological Satellite Center of Japan Meteorological Agency, 3-235, Kiyose, Nakakiyoto, Tokyo 204-0012, Japan

E-mail: hidehiko.murata@met.kishou.go.jp

J-stage Advance Published Date: 13 July 2018 $\mu \mathrm{m}$ ), and B01 (blue: $0.47 \mu \mathrm{m}$ ) enables the production of true color imagery.

True color imagery (e.g., Miller et al. 2012) is intended to be as seen by the human eye. It is produced via a combination of visible bands whose central wavelengths are near the three primary colors of visible light (red, green, and blue). It was previously produced from sensors on polar-orbiting satellite platforms, such as the Moderate Resolution Imaging Spectroradiometer (MODIS) aboard the National Aeronautics and Space Administration's (NASA) Terra and Aqua satellites and the Visible Infrared Imaging Radiometer Suite (VIIRS) aboard the Suomi National Polar-Orbiting Partnership (Suomi-NPP) satellite 
Table 1. Central wavelengths of Himawari-8 and -9 AHI bands in $\mu \mathrm{m}$. Based on JMA/MSC website information at http://www.data.jma.go.jp/mscweb/en/himawari89/ space_segment/spsg_ahi.html.

\begin{tabular}{ccc}
\hline Band & Himawari-8/AHI & Himawari-9/AHI \\
\hline B01 & 0.47 & 0.47 \\
B02 & 0.51 & 0.51 \\
B03 & 0.64 & 0.64 \\
B04 & 0.86 & 0.86 \\
B05 & 1.6 & 1.6 \\
B06 & 2.3 & 2.3 \\
B07 & 3.9 & 3.8 \\
B08 & 6.2 & 6.2 \\
B09 & 6.9 & 7.0 \\
B10 & 7.3 & 7.3 \\
B11 & 8.6 & 8.6 \\
B12 & 9.6 & 9.6 \\
B13 & 10.4 & 10.4 \\
B14 & 11.2 & 11.2 \\
B15 & 12.4 & 12.4 \\
B16 & 13.3 & 13.3 \\
\hline
\end{tabular}

operated by NASA and the National Oceanic and Atmospheric Administration (NOAA).

True color imagery aids intuitive understanding of imagery by coloring features such as vegetation, deserts, snow cover, coral reefs, river sediment discharge, and phytoplankton blooms. Atmospheric phenomena, such as volcanic ashes, pollution, biomass smoke, and dust storms, can also be distinguished readily from meteorological white clouds in true color. The imag- ery even facilitates the discrimination of white clouds against color-diverse backgrounds.

Geostationary platforms offer frequent observations, enabling detailed monitoring of temporal changes in atmospheric features, whereas polar-orbiting platforms are limited to only a few observations a day. In addition, frequent observations increase surface observation opportunities via the accumulation of cloud-free areas.

The simplest way to display true color imagery on a computer screen is to compose red, green, and blue band data as RGB primary colors. The simplest true color imagery provides a certain level of realistic coloring but may not be accurate because the RGB primary colors of output devices (e.g., liquid crystal displays) differ from those of input devices (e.g., satellite sensors). Additionally, spectral response functions (SRFs), which differ among satellite sensors, may cause color differences.

True color imagery produced via a simple composition of the AHI's native RGB bands (red: B03 (0.64 $\mu \mathrm{m})$; green: B02 $(0.51 \mu \mathrm{m})$; blue: B01 $(0.47 \mu \mathrm{m}))$ is shown in Fig. 1a. Although the imagery may superficially appear to have a certain level of realistic coloring, unnatural colors such as dark tropical vegetation and overly red deserts are present.

Miller et al. (2016) reported that the colors of vegetation and desert areas in AHI's native RGB true color imagery deviate from those of the MODIS Blue Marble dataset (Stöckli et al. 2007). In order to mitigate the difference, the AHI green band is modified to form a hybrid green band that boosts vegetation/desert
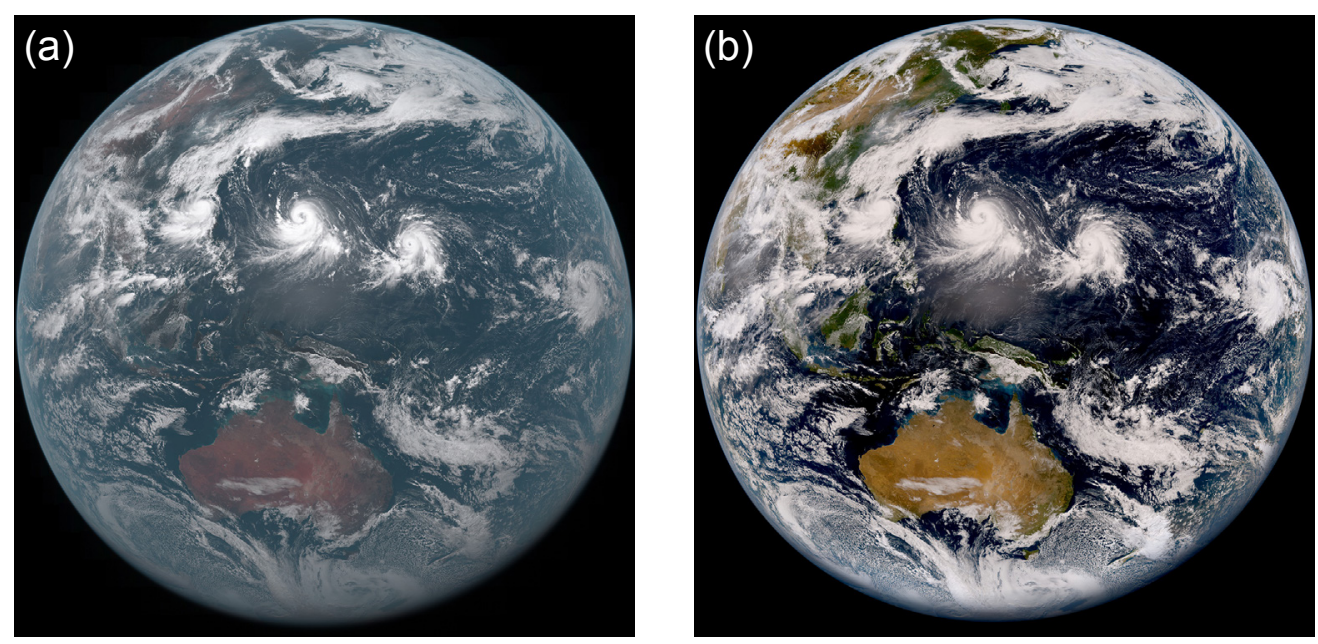

Fig. 1. Himawari-8 full-disk true color imagery (7 July 2015, 0300 UTC). (a) Simple composition of AHI's native RGB bands (enhanced with gamma correction, $\Gamma=2.0$ ). (b) TCR imagery. 
signals via mixing of B02 $(0.51 \mu \mathrm{m})$ and B04 (0.86 $\mu \mathrm{m})$. This significantly improves the resulting true color imagery.

In this study, we aimed to reproduce colors seen by the human eye instead of those in existing true color imagery (e.g., MODIS and VIIRS), which may vary with differences in primary colors as mentioned above. For the purpose of color reproduction, an approach based on the International Commission on Illumination (CIE) 1931 XYZ color system (CIE 1932) was applied. This color system is standard for quantitative expression of colors seen by the human eye and represents any color using XYZ tristimulus values regardless of devices. In the approach, the RGB signals obtained by a satellite sensor are converted into XYZ values, which are then reconverted to RGB signals for output devices to reproduce colors.

Accurate XYZ values obtained from satellite observations enable the faithful reproduction of colors as seen by the human eye regardless of satellite sensors. In this study, we examined a $3 \times 3$ conversion matrix that converts satellite observations (RGB signals) into $X Y Z$ values with a good accuracy. The accuracy of the obtained XYZ values was evaluated by an objective technique using a number of hyperspectral reflectance spectra for various types of earth surfaces. The technique is also utilized in consideration of improving the accuracy of the obtained XYZ values.

Output devices were assumed to comply with sRGB (IEC 61966-2-1: IEC 1999), which is adopted as an international standard for color space in many common electronic devices such as display units and printers. Conversion from XYZ values to RGB signals for output devices is performed appropriately by a given $3 \times 3$ matrix for the standard and is not discussed in this study.

This color reproduction approach is applicable to other satellites with different band configurations, in particular to new-generation geostationary satellites such as GOES-16 (Schmit et al. 2005, 2008, 2017), FY-4A (Yang et al. 2017), GEO-KOMPSAT-2A (Griffith and Yang 2016) and MTG (Mohr 2014). It can potentially be developed to provide a standard approach for the rendering of true color imagery with a uniform appearance regardless of satellite sensors in order to provide consistency for users.

True color imagery produced using this color reproduction approach (termed True Color Reproduction (TCR) imagery) also involves the application of Rayleigh scattering, atmospheric correction, and loglinear scaling (Miller et al. 2016) via techniques provided by the Cooperative Institute for Research in the
Atmosphere (CIRA) at Colorado State University, USA.

Figure $1 \mathrm{~b}$ shows TCR imagery for the same time as the imagery in Fig. 1a. Land surface colors are dramatically improved and appear more realistic. The removal of Rayleigh scattering clarifies the imagery, whereas the simple composition in Fig. 1a has a milky-blue appearance.

The Meteorological Satellite Center (MSC) began providing online TCR imagery on 10 May 2016 (Himawari Real-Time Image; http://www.data.jma. go.jp/mscweb/data/himawari/). TCR imagery was also utilized for the first images of Himawari-9, which was launched on 2 November 2016 (Japan Meteorological Agency 2017).

This article details the steps taken to produce the TCR imagery as follows. Section 2 defines the basic terms of the CIE 1931 XYZ color system. Section 3 describes the matrices used for conversion between RGB signals and XYZ values. Section 4 outlines a technique for the evaluation of $\mathrm{XYZ}$ values obtained from satellite data. Section 5 highlights the reconstruction of the RGB band combination, especially for green band modification. Section 6 presents the TCR imagery production procedure. Section 7 provides a discussion of related case studies and other matters. Finally, Section 8 gives the conclusions.

\section{CIE 1931 XYZ color system}

In this section, we will define the basic terms used in this study in relation to the CIE $1931 \mathrm{XYZ}$ color system. For further details, see other references (e.g., Ohta 1997; Taniguchi and Zhang 2012; Giorgianni and Madden 2008).

The CIE 1931 XYZ color system is a standard for the quantitative expression of any color using XYZ tristimulus values independently from standards and devices (CIE 1932). The XYZ values for a color stimulus with a spectral distribution $\varphi(\lambda)$ are determined as follows:

$$
\begin{aligned}
& X=k \int_{v i s} \varphi(\lambda) \cdot \bar{x}(\lambda) d \lambda, \\
& Y=k \int_{v i s} \varphi(\lambda) \cdot \bar{y}(\lambda) d \lambda, \\
& Z=k \int_{v i s} \varphi(\lambda) \cdot \bar{z}(\lambda) d \lambda,
\end{aligned}
$$

where $\lambda$ is the wavelength and $\int_{v i s}$ represents integration in the visible wavelength range. $\bar{x}(\lambda), \bar{y}(\lambda)$, and $\bar{z}(\lambda)$ are color matching functions, and the CIE 1931 two-degree color matching functions (Fig. 2) were applied in this study. The constant $k$ is used to 


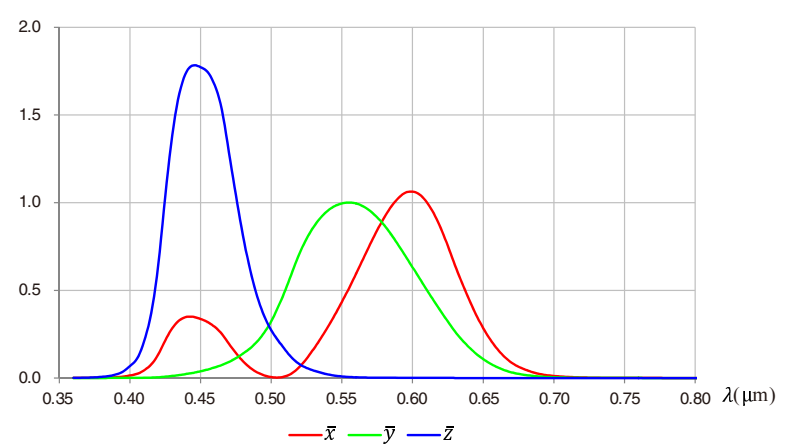

Fig. 2. CIE 1931 two-degree color matching functions. red line: $\bar{x}(\lambda)$; green line: $\bar{y}(\lambda)$; blue line: $\bar{z}(\lambda)$. The horizontal axis represents the wavelength $\lambda(\mu \mathrm{m})$. Data from Colour \& Vision Research laboratory and database (http://cvrl.ioo. ucl.ac.uk/).

scale $Y$ as luminance; it did not need to be determined in this study because only relative values of XYZ were required. Colors with the same $\mathrm{XYZ}$ values are perceived as identical by the human eye (i.e., the CIE standard observer) even if the spectral distributions $\varphi(\lambda)$ are different.

The chromaticity coordinates of the XYZ color system, described as $(x, y)$, are intersection points of the color vector $(X, Y, Z)$ and the unit plane $X+Y+$ $Z=1$ in an XYZ three-dimensional space. The chromaticity coordinates (including $z$, which depends on $x$ and $y$ ) are calculated as follows:

$$
\begin{aligned}
& x=\frac{X}{X+Y+Z}, \\
& y=\frac{Y}{X+Y+Z}, \\
& z=\frac{Z}{X+Y+Z} .
\end{aligned}
$$

A representation of chromaticity coordinates on a plane is called a chromaticity diagram (Fig. 3). The inside of the horseshoe-shaped area demarcates the visible range for human vision, and the colors approximately correspond to the chromaticity coordinates. The outer curve corresponds to pure (monochromatic) colors, and the area near the point $(x, y)=\left(\frac{1}{3}, \frac{1}{3}\right)$ corresponds to white (or achromatic colors).

\section{Matrices for conversion between RGB and XYZ}

To support the reproduction of colors as seen by the human eye from satellite observations, a color reproduction approach based on the CIE XYZ color system

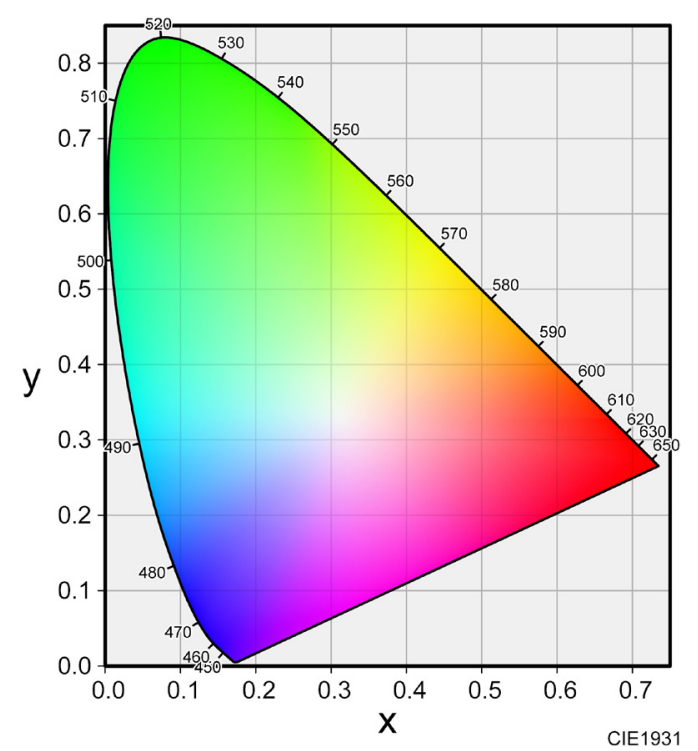

Fig. 3. CIE 1931 xy chromaticity diagram (drawn with Chromaticity Diagram Maker ColorAC, http://n-colorspace.cool.coocan.jp/index.html). The numbers on the outer curve denote the wavelength of the corresponding monochromatic light (nm).

was adopted in this study. With the approach, RGB signals (scaled radiance or reflectance) observed by a satellite are converted to $\mathrm{XYZ}$ values, which are then reconverted into RGB signals for output devices. XYZ values with a good accuracy enable faithful color reproduction. The approach enables the consideration of differences in RGB primary colors among satellites and output devices, which cause differences in the reproduced color. Figure 4 shows a conceptual representation of the color reproduction approach. Conversion from satellite RGB signals to $X Y Z$ values and from $X Y Z$ values to output RGB signals is performed with the application of a $3 \times 3$ conversion matrix (or inverse matrix) generated as described below.

RGB signals for devices (e.g., satellite sensors, displays) are converted to XYZ values using the following matrix:

$$
\left(\begin{array}{c}
X \\
Y \\
Z
\end{array}\right)=\left(\begin{array}{ccc}
X_{R} & X_{G} & X_{B} \\
Y_{R} & Y_{G} & Y_{B} \\
Z_{R} & Z_{G} & Z_{B}
\end{array}\right)\left(\begin{array}{l}
R \\
G \\
B
\end{array}\right),
$$

where $\left(X_{R}, Y_{R}, Z_{R}\right)$ are the $\mathrm{XYZ}$ (tristimulus) values of red alone and $\left(X_{G}, Y_{G}, Z_{G}\right)$ and $\left(X_{B}, Y_{B}, Z_{B}\right)$ are the same for green and blue, respectively. The values of 


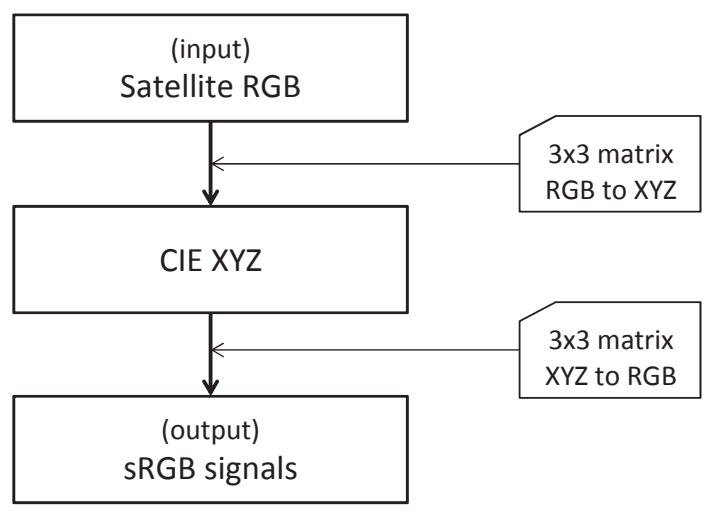

Fig. 4. Conceptual representation of color reproduction.

Table 2. Chromaticity coordinate symbols.

\begin{tabular}{ccccc}
\hline $\begin{array}{c}\text { Chromaticity } \\
\text { coordinates }\end{array}$ & Red & Green & Blue & White \\
\hline$x$ & $x_{R}$ & $x_{G}$ & $x_{B}$ & $x_{W}$ \\
$y$ & $y_{R}$ & $y_{G}$ & $y_{B}$ & $y_{W}$ \\
$z=1-x-y$ & $z_{R}$ & $z_{G}$ & $z_{B}$ & $z_{W}$ \\
\hline
\end{tabular}

the input signals $R, G$, and $B$ vary between 0 and 1 . $\mathrm{XYZ}$ values are converted into RGB signals reversibly using the inverse matrix.

The matrix components are calculated by substituting the chromaticity coordinates of each RGB primary color and the white point (shown in Table 2) as follows:

$$
\left(\begin{array}{ccc}
X_{R} & X_{G} & X_{B} \\
Y_{R} & Y_{G} & Y_{B} \\
Z_{R} & Z_{G} & Z_{B}
\end{array}\right)=\left(\begin{array}{lll}
x_{R} & x_{G} & x_{B} \\
y_{R} & y_{G} & y_{B} \\
z_{R} & z_{G} & z_{B}
\end{array}\right)\left(\begin{array}{ccc}
S_{R} & 0 & 0 \\
0 & S_{G} & 0 \\
0 & 0 & S_{B}
\end{array}\right),
$$

where

$$
\left(\begin{array}{l}
S_{R} \\
S_{G} \\
S_{B}
\end{array}\right)=\left(\begin{array}{lll}
x_{R} & x_{G} & x_{B} \\
y_{R} & y_{G} & y_{B} \\
z_{R} & z_{G} & z_{B}
\end{array}\right)^{-1}\left(\begin{array}{c}
\frac{x_{w}}{y_{w}} \\
1 \\
\frac{z_{w}}{y_{w}}
\end{array}\right)
$$

The matrix derivation is detailed in the Appendix.

\subsection{AHI conversion matrix}

To generate the conversion matrix described above, the chromaticity coordinates in Table 2 need to be obtained for a combination of satellite RGB bands. The

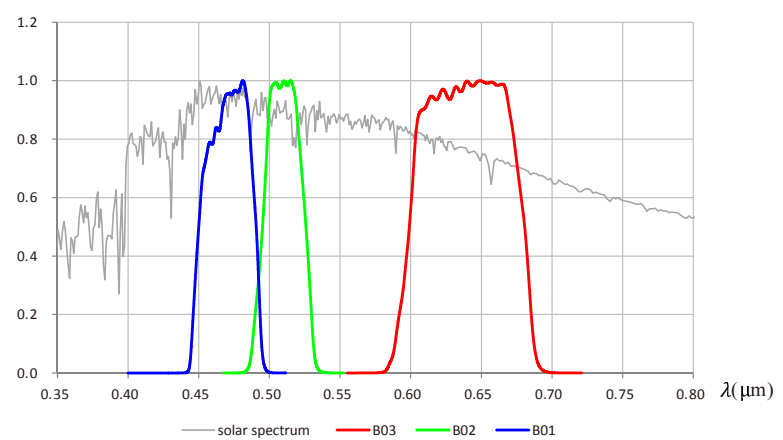

Fig. 5. SRFs of AHI/B01 to B03 (red: B03; green: B02; blue: B01) and the normalized solar spectrum (ASTM E-490-00) (gray). AHI SRFs are from the JMA/MSC website (http://www.data. jma.go.jp/mscweb/en/himawari89/space_segment/ spsg_ahi.html).

relative $\mathrm{XYZ}$ values for satellite bands are obtained using color matching functions (Fig. 2) as with Eq. (1) as follows:

$$
\begin{aligned}
X^{\prime} & =\int_{v i s} S(\lambda) P(\lambda) \bar{x}(\lambda) d \lambda, \\
Y^{\prime} & =\int_{v i s} S(\lambda) P(\lambda) \bar{y}(\lambda) d \lambda, \\
Z^{\prime} & =\int_{v i s} S(\lambda) P(\lambda) \bar{z}(\lambda) d \lambda,
\end{aligned}
$$

where $S(\lambda)$ is the SRF of the band and $P(\lambda)$ is the solar spectrum. $X^{\prime} Y^{\prime} Z^{\prime}$ represents relative $X Y Z$ values to distinguish them from the absolute $X Y Z$ values in Eq. (1).

Figure 5 shows the SRFs of AHI's native RGB bands for application in Eq. (6). For the solar spectrum $P(\lambda)$, the 2000 American Society for Testing \& Materials (ASTM) Standard Extraterrestrial Solar Spectrum Reference E-490-00 (ASTM 2000) is utilized (also shown in Fig. 5). The solar spectrum is sufficient as relative values because it is scaled. Seasonal variations in solar irradiance are not necessary to be considered for the same reason.

The chromaticity coordinates are obtained as per Eq. (2) as follows:

$$
\begin{aligned}
& x=\frac{X^{\prime}}{X^{\prime}+Y^{\prime}+Z^{\prime}}, \\
& y=\frac{Y^{\prime}}{X^{\prime}+Y^{\prime}+Z^{\prime}}, \\
& z=\frac{Z^{\prime}}{X^{\prime}+Y^{\prime}+Z^{\prime}} .
\end{aligned}
$$


Table 3. Chromaticity coordinates of AHI visible bands and the white point.

\begin{tabular}{ccccc}
\hline $\begin{array}{c}\text { Chromaticity } \\
\text { coordinates }\end{array}$ & $\begin{array}{c}\text { Red } \\
(\mathrm{AHI} / \mathrm{B} 03)\end{array}$ & $\begin{array}{c}\text { Green } \\
(\mathrm{AHI} / \mathrm{B} 02)\end{array}$ & $\begin{array}{c}\text { Blue } \\
(\mathrm{AHI} / \mathrm{B} 01)\end{array}$ & $\begin{array}{c}\text { White } \\
\text { (Sunlight) }\end{array}$ \\
\hline$x$ & 0.6789 & 0.0456 & 0.1252 & 0.3236 \\
$y$ & 0.3209 & 0.7046 & 0.0702 & 0.3326 \\
$z=1-x-y$ & 0.0002 & 0.2498 & 0.8046 & 0.3438 \\
\hline
\end{tabular}

The obtained AHI native RGB band chromaticity coordinates corresponding to those in Table 2 are summarized in Table 3.

The white point chromaticity coordinates (also shown in Table 3) are obtained from the following $\mathrm{X}^{\prime} \mathrm{Y}^{\prime} \mathrm{Z}^{\prime}$ values using Eq. (7) in the same way:

$$
\begin{aligned}
X^{\prime} & =\int_{v i s} P(\lambda) \bar{x}(\lambda) d \lambda, \\
Y^{\prime} & =\int_{v i s} P(\lambda) \bar{y}(\lambda) d \lambda, \\
Z^{\prime} & =\int_{v i s} P(\lambda) \bar{z}(\lambda) d \lambda .
\end{aligned}
$$

The conversion matrix in Eq. (3) for the combination of AHI native RGB bands is obtained by applying the chromaticity coordinates shown in Table 3 to Eqs. (4) and (5) as follows:

$$
\left(\begin{array}{ccc}
X_{R_{A H I}} & X_{G_{A H I}} & X_{B_{A H I}} \\
Y_{R_{A H I}} & Y_{G_{A H I}} & Y_{B_{A H I}} \\
Z_{R_{A H I}} & Z_{G_{A H I}} & Z_{B_{A H I}}
\end{array}\right)=\left(\begin{array}{lll}
0.8068 & 0.0353 & 0.1307 \\
0.3813 & 0.5454 & 0.0733 \\
0.0002 & 0.1934 & 0.8400
\end{array}\right),
$$

where the subscripts of $\mathrm{XYZ}$ indicate primary color and devices; for example, $X_{R_{A H I}}$ represents the $\mathrm{X}$ tristimulus value of the AHI's red (R) band.

\section{$3.2 s R G B$ conversion matrix}

The XYZ values obtained via conversion from satellite RGB signals are reconverted by applying another $3 \times 3$ matrix to RGB signals for output devices, and the colors are then reproduced on the relevant device. In this study, it was assumed that output devices are compliant with sRGB, which is an international standard for color space defined by the International Electrotechnical Commission (IEC) (IEC 61966-2-1: IEC 1999) and adopted in many common electronic devices such as displays and printers. D65, a standard illuminant defined by the CIE, was adopted as the white point. The chromaticity coordinates for sRGB and D65 are summarized in Table 4.

The conversion matrix for sRGB is obtained as per

\begin{tabular}{|c|c|c|c|c|}
\hline $\begin{array}{l}\text { Chromaticity } \\
\text { coordinates }\end{array}$ & Red & Green & Blue & $\begin{array}{l}\text { White } \\
\text { (D65) }\end{array}$ \\
\hline$x$ & 0.64 & 0.30 & 0.15 & 0.3127 \\
\hline$y$ & 0.33 & 0.60 & 0.06 & 0.3290 \\
\hline$z=1-x-y$ & 0.03 & 0.10 & 0.79 & 0.3583 \\
\hline$\left(X_{R_{s R G B}} X_{G_{s R C}}\right.$ & $X_{B_{S R G B}}$ & 0.4124 & 0.3576 & 0.1805 \\
\hline$Y_{R_{s R G B}} \quad Y_{G_{s R G G}}$ & $Y_{B_{s R C B}}$ & $=0.2126$ & 0.7152 & 0.0722 \\
\hline$Z_{R_{s R G B}} Z_{G_{S R G}}$ & $Z_{B_{s R G B}}$ & 0.0193 & 0.1192 & 0.9505 \\
\hline
\end{tabular}
Eq. (9) by applying the given chromaticity coordinates shown in Table 4 as follows:
Table 4. sRGB and D65 chromaticity coordinates.

The inversion matrix for conversion from XYZ to RGB is shown below:

$$
\begin{aligned}
& \left(\begin{array}{ccc}
X_{R_{S R G B}} & X_{G_{S R G B}} & X_{B_{s R G B}} \\
Y_{R_{S R G B}} & Y_{G_{s R G B}} & Y_{B_{S R G B}} \\
Z_{R_{s R G B}} & Z_{G_{S R G B}} & Z_{B_{S R G B}}
\end{array}\right)^{-1} \\
& =\left(\begin{array}{rrr}
3.2406 & -1.5372 & -0.4986 \\
-0.9689 & 1.8758 & 0.0415 \\
0.0557 & -0.2040 & 1.0570
\end{array}\right) \text {. }
\end{aligned}
$$

\subsection{Remapping matrix}

The chromaticity coordinates for AHI and sRGB summarized in Tables 3 and 4 are plotted in a chromaticity diagram (Fig. 6). The triangles connecting the chromaticity coordinates of RGB primary colors are called the color gamut and indicate the range of colors that can be expressed by mixing RGB primary colors.

Color gamut differences cause corresponding variations in reproduced colors. For example, a color with $\mathrm{RGB}=(1,0,0)$ obtained by the AHI corresponds to the point $\mathrm{R}: \mathrm{AHI} / \mathrm{B} 03$ in the diagram, but to R:sRGB when the signal is used as that of sRGB. As mentioned earlier, in order to mitigate color variations caused by primary color differences, RGB signals obtained by the AHI are converted into XYZ values and then reconverted to RGB signals for the sRGB standard.

The direct conversion of RGB signals from $\mathrm{AHI}$ to sRGB is performed using the product matrix (referred 


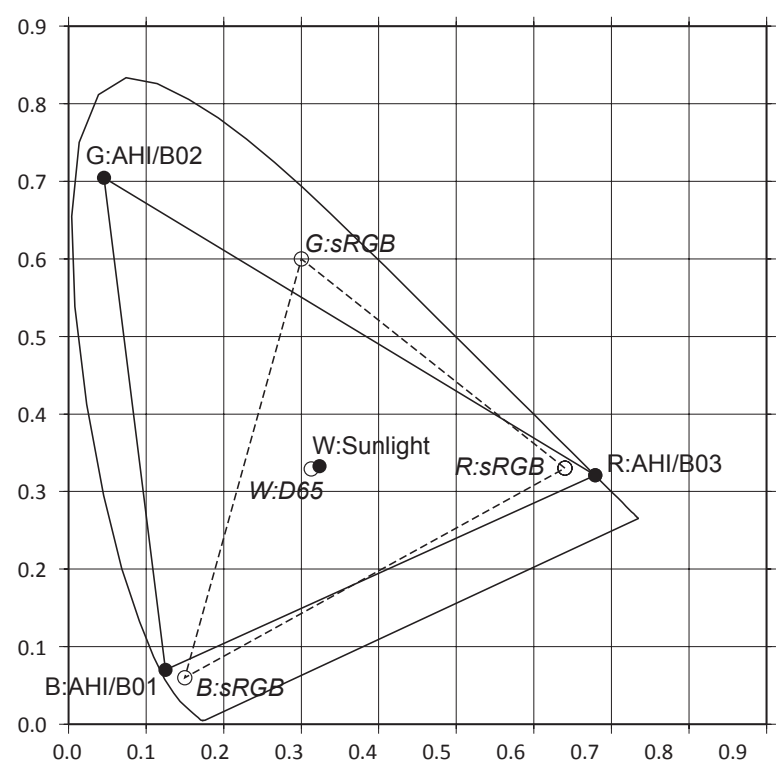

Fig. 6. Chromaticity diagram with the chromaticity coordinates of RGB (color gamut) and white point for the AHI and sRGB. Solid lines and filled circles: AHI RGB and white point (sunlight). Dashed lines and white circles: sRGB and D65.

to here as the remapping matrix) for the two matrices (i.e., XYZ to RGB (11) and RGB to XYZ (9)) as shown in the following equation:

$$
\begin{aligned}
& \left(\begin{array}{l}
R_{s R G B} \\
G_{s R G B} \\
B_{s R G B}
\end{array}\right)=\left(\begin{array}{ccc}
X_{R_{s R G B}} & X_{G_{s R G B}} & X_{B_{s R G B}} \\
Y_{R_{S R G B}} & Y_{G_{S R G B}} & Y_{B_{S S G B}} \\
Z_{R_{S R G B}} & Z_{G_{S R G B}} & Z_{B_{s R G B}}
\end{array}\right)^{-1}\left(\begin{array}{c}
X \\
Y \\
Z
\end{array}\right) \\
& =\left(\begin{array}{ccc}
X_{R_{S R G B}} & X_{G_{S R C B}} & X_{B_{S S C G B}} \\
Y_{R_{S R G B}} & Y_{G_{S R C B}} & Y_{B_{S R R B}} \\
Z_{R_{S R G B}} & Z_{G_{S R G B}} & Z_{B_{S R G B}}
\end{array}\right)^{-1} \\
& \times\left(\begin{array}{ccc}
X_{R_{A H I}} & X_{G_{A H I}} & X_{B_{A H I}} \\
Y_{R_{A H I}} & Y_{G_{A H I}} & Y_{B_{A H I}} \\
Z_{R_{A H I}} & Z_{G_{A H I}} & Z_{B_{A H I}}
\end{array}\right)\left(\begin{array}{l}
R_{A H I} \\
G_{A H I} \\
B_{A H I}
\end{array}\right) \\
& =\left(\begin{array}{rrr}
2.0283 & -0.8204 & -0.1080 \\
-0.0665 & 0.9969 & 0.0457 \\
-0.0326 & 0.0951 & 0.8802
\end{array}\right)\left(\begin{array}{l}
R_{A H I} \\
G_{A H I} \\
B_{A H I}
\end{array}\right) \text {. }
\end{aligned}
$$

Figure 7 shows true color imageries for the same time as in Fig. 1. Figure 7a shows that of the AHI native RGB bands, and Fig. 7b is based on the application of the remapping matrix shown in Eq. (12). Unexpectedly, the colors in Fig. $7 \mathrm{~b}$ are clearly less natural than those of the simple composition (Fig. 7a). Accordingly, it is suspected that the matrix (9) does not convert the RGB signals into XYZ values appropriately.

\section{Evaluation of the obtained $\mathrm{XYZ}$ values}

An objective technique to evaluate the accuracy of the $\mathrm{XYZ}$ values obtained from satellite RGB signals has been developed in order to support the exploration and handling of causes behind the unnatural colors described in the previous section. This technique also allows the evaluation of the colors of the simple native RGB band composition.

Figure 8 outlines the evaluation. Accurate XYZ values for materials with known reflectance spectra can be directly derived using color matching functions (shown in the left-hand part of the chart). Conversely, in the right-hand part, XYZ values are obtained by applying the conversion matrix to simulated satellite RGB data generated using satellite band SRFs. The obtained XYZ values are evaluated in comparison to the accurate $X Y Z$ values.

\subsection{Datasets for evaluation}

The datasets and procedures shown in Fig. 8 are described below.

\section{(i) Reflectance spectra dataset}

A number of hyperspectral reflectance spectra are used as a source dataset for evaluation. All reflectance spectra come from the United States Geological Survey (USGS) Digital Spectral Library (Clark et al. 2007). The library ranges from ultraviolet to far-infrared wavelengths (from 0.2 to $150 \mu \mathrm{m}$ ) with around 1,000 data types for the visible to nearinfrared spectral region, and it includes data on natural materials, such as minerals, soils, open water, snow/ice, and vegetation, and on artificial materials, such as paints and plastics.

The reflectance spectra of natural materials expected to be present within the satellite coverage area are selected from the library based on the criteria that there should be no large gaps and there should be sufficient coverage of the necessary spectral region from visible to near infrared.

As part of the dataset, 126 material types selected by Vidot and Borbás (2014), who generated Bidirectional Reflectance Distribution Function (BRDF) maps for the RTTOV-11 radiative transfer model, are utilized. The selection includes 26 spectra from Chapter V (Plants, Vegetation Communities, Mixtures with Vegetation, and Microorganisms) (referred 

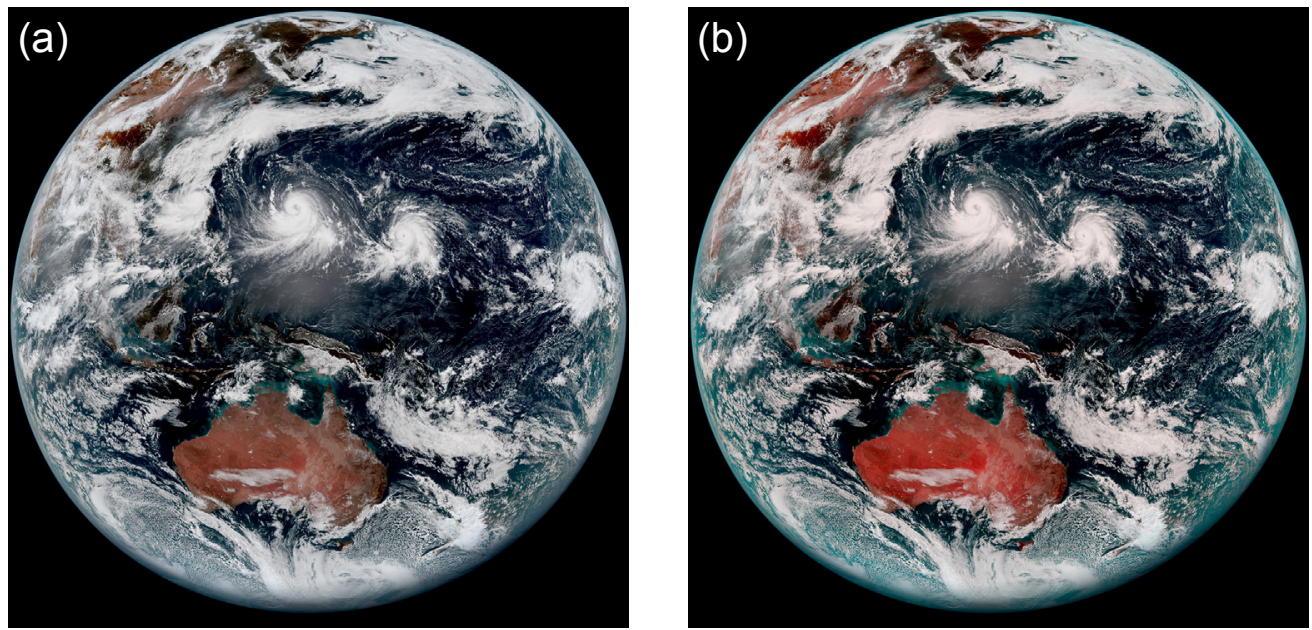

Fig. 7. Himawari-8 full-disk true color imagery for the same time as Fig. 1. (a) Simple composition of AHI native RGB bands. (b) rendering with the remapping matrix (12). Rayleigh scattering correction and log-linear scaling (Section 6) are applied to both.

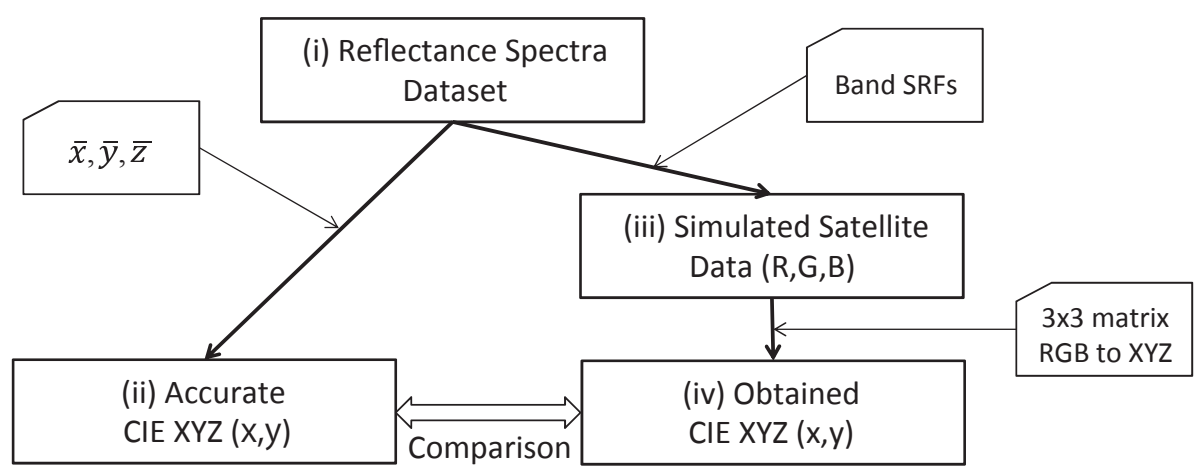

Fig. 8. Evaluation flowchart.

to here simply as vegetation) and 100 spectra from Chapter S (Soils, Rocks, and Mixtures) (referred to here as soils). In addition to the selection, 23 spectra from Chapter L (Liquids, Liquid Mixtures, Water, Other Volatiles, and Frozen Volatiles) (referred to here as liquids) are included and, for a total of 149 spectra, are applied to this evaluation (Fig. 9).

Although the dataset might not cover every type of material on the earth's surface, it was assumed to incorporate a large enough variety of surface types. It was also assumed that satellite pixels, including multiple surface types, will exhibit intermediate characteristics of their constituents and that the obtained XYZ values for such pixels will be accurate if the corresponding values for the individual constituent types are also accurate.

\section{(ii) Accurate CIE XYZ values}

Accurate XYZ values for materials with known reflectance spectra can be directly derived using the color matching functions shown in Fig. 2. The $\mathrm{X}^{\prime} \mathrm{Y}^{\prime} \mathrm{Z}^{\prime}$ (XYZ relative values) of a certain reflecting material are obtained as with Eq. (1) as follows:

$$
\begin{aligned}
X^{\prime} & =\int_{v i s} R(\lambda) P(\lambda) \bar{x}(\lambda) d \lambda, \\
Y^{\prime} & =\int_{v i s} R(\lambda) P(\lambda) \bar{y}(\lambda) d \lambda, \\
Z^{\prime} & =\int_{v i s} R(\lambda) P(\lambda) \bar{z}(\lambda) d \lambda,
\end{aligned}
$$

where $R(\lambda)$ is the reflectance spectrum of the material and $P(\lambda)$ is the solar spectrum. Chromaticity coordinates $(x, y)$ are obtained using Eq. (7). Figure 

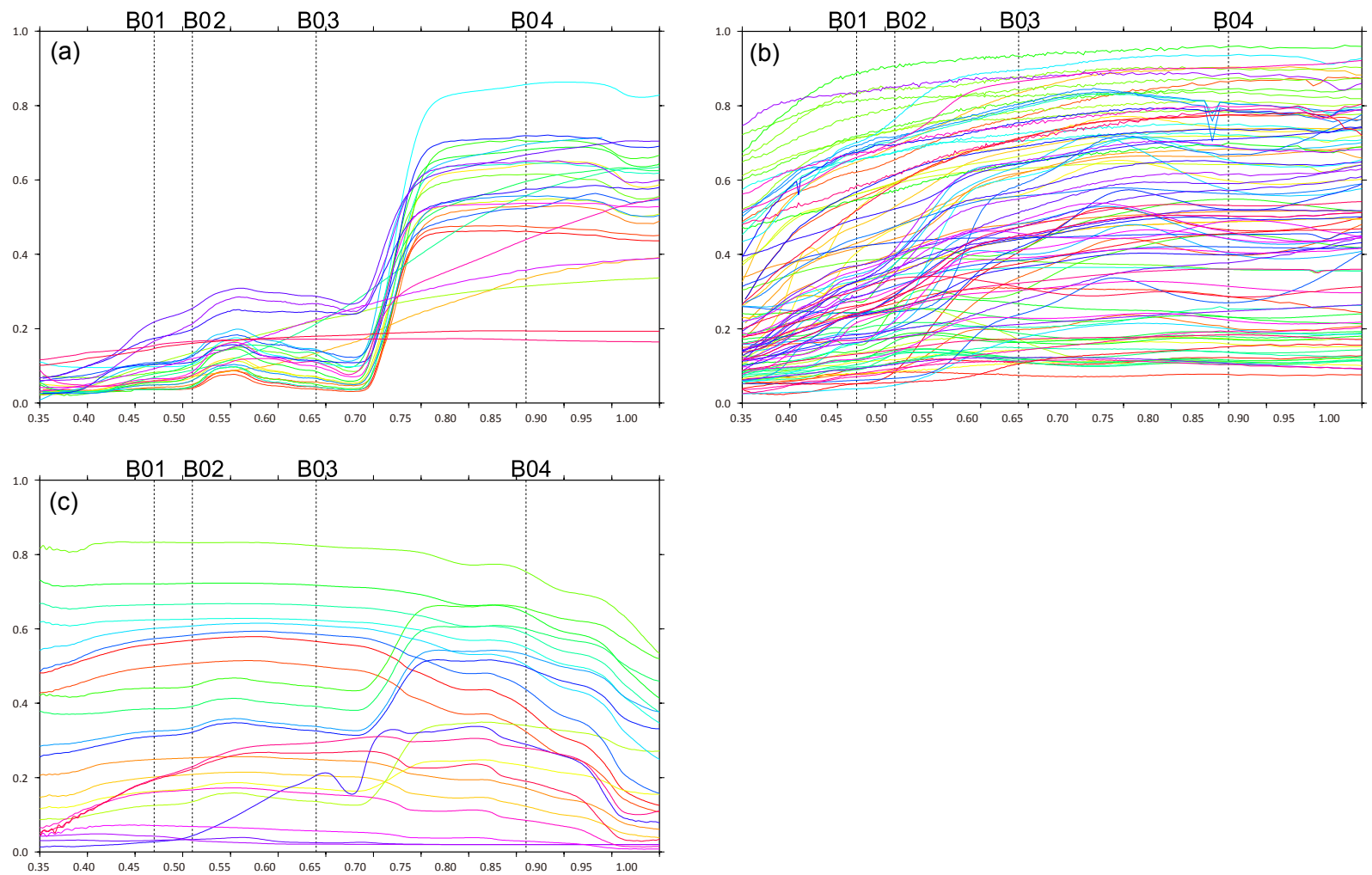

Fig. 9. Reflectance spectra from the UGSG Digital Spectral Library (Clark et al. 2007). (a) Vegetation (plants, vegetation communities, mixtures with vegetation). (b) Soils (soils, rocks, and mixtures). (c) Liquids (liquid mixtures, water, other volatiles, and frozen volatiles). Horizontal axis: wavelength $(\mu \mathrm{m})$; vertical axis: reflectance. Vertical lines correspond to the central wavelengths of AHI bands (B01 to B04).

10a shows the distribution of accurate chromaticity coordinates for the selected spectrum dataset. See Fig. 3 for information on the approximate correspondence between chromaticity coordinates and related colors in the chromaticity diagram.

Absolute XYZ values are obtained by scaling the relative $\mathrm{X}^{\prime} \mathrm{Y}^{\prime} \mathrm{Z}^{\prime}$ values with $Y_{w}$, which is the luminance for the material of $R(\lambda)=1$, as follows:

$$
\begin{aligned}
Y_{w} & =\int_{v i s} P(\lambda) \bar{y}(\lambda) d \lambda, \\
X & =X^{\prime} / Y_{w}, \\
Y & =Y^{\prime} / Y_{w}, \\
X & =Z^{\prime} / Y_{w} .
\end{aligned}
$$

The XYZ values can be converted into sRGB signals by applying the conversion matrix (11), and accurate colors are then reproduced on SRGB devices (i.e., interpretation from XYZ values to colors).
Figure 10b shows a color palette with accurate colors for the selected spectra. In the color palette, signals are enhanced with log-linear scaling (Section 6) as applied to satellite imagery.

(iii) Simulated satellite data

Simulated satellite data expressed in reflectance $R_{\text {sim }}$ for a reflectance spectrum are obtained as follows:

$$
R_{s i m}=\frac{\int S(\lambda) R(\lambda) P(\lambda) d \lambda}{\int S(\lambda) P(\lambda) d \lambda}
$$

where $S(\lambda)$ is the SRF of the satellite band, $R(\lambda)$ is the reflectance spectrum of the material, and $P(\lambda)$ is the solar spectrum. This is a normalized value that varies between 0 and 1 . Only simple reflection at the surface is considered, and no atmospheric effects are taken into account. 

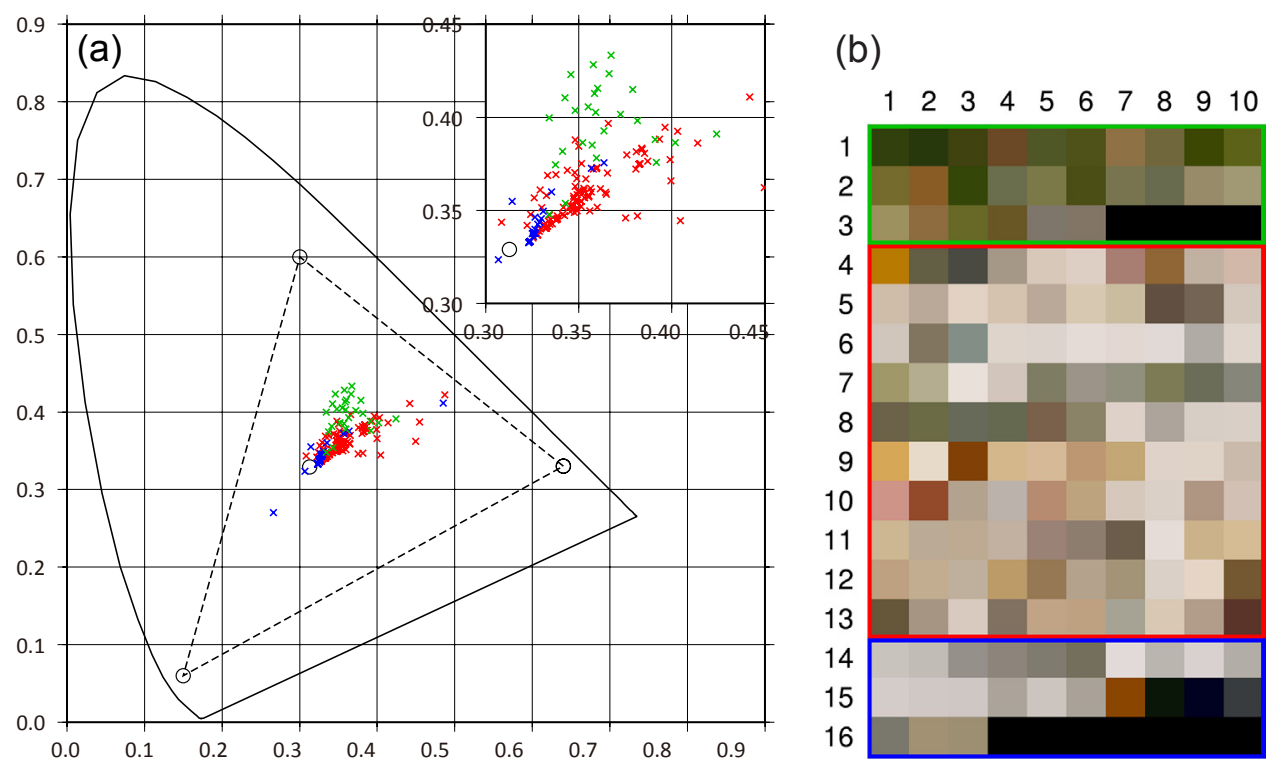

Fig. 10. Accurate chromaticity coordinates and corresponding colors. (a) Distribution of accurate chromaticity coordinates. The rectangular area to the upper right is an enlarged view of the center of the diagram, where the plots are dense. Plot colors correspond to the library chapter (green: vegetation; red: soils; blue: liquids). The sRGB color gamut and D65 are also shown by the dashed triangle and the white circles. (b) Color palette with accurate colors corresponding to the plots in (a). Frame colors correspond to the relevant library chapter as per (a).

(iv) Obtained CIE XYZ values

$\mathrm{XYZ}$ values to be evaluated are obtained by applying the conversion matrix to the simulated satellite data above.

\subsection{Comparison between accurate and obtained XYZ values}

The results of comparison between accurate XYZ values and those obtained are outlined below for two cases.

\section{a. Simple composition of $A H I$ native $R G B$ bands}

First, the XYZ values of the simple composition of AHI native RGB bands requiring improvement are evaluated using this technique as a start point before the evaluation of the XYZ values obtained via conversion matrix (9) application. For this case, satellite RGB signals are assumed to be used as those of sRGB. XYZ values are then obtained by applying matrix (10) as follows:

$$
\left(\begin{array}{c}
X \\
Y \\
Z
\end{array}\right)=\left(\begin{array}{ccc}
X_{R_{s R G B}} & X_{G_{s R G B}} & X_{B_{s R G B}} \\
Y_{R_{S R G B}} & Y_{G_{S R G B}} & Y_{B_{s R G B}} \\
Z_{R_{S R G B}} & Z_{G_{s R G B}} & Z_{B_{s R G B}}
\end{array}\right)\left(\begin{array}{c}
R_{A H I} \\
G_{A H I} \\
B_{A H I}
\end{array}\right) .
$$

The simulated data of the AHI native RGB bands described above are applied as $\left(R_{A H I}, G_{A H I}, B_{A H I}\right)$, and the chromaticity coordinates are obtained from the XYZ values using Eq. (2).

A comparison of accurate and obtained chromaticity coordinates is shown in Fig. 11a. Colored plots indicate the individual accurate chromaticity coordinates shown in Fig. 10a, and arrows starting from plots point at the obtained chromaticity. Arrow lengths indicate discrepancies between accurate and obtained chromaticity coordinates, with shorter values indicating a better XYZ accuracy. Generally, the arrows are not short and this means the colors are less accurate.

Focusing on plots for vegetation (shown in green) in the vicinity of (A), the arrow tips are closer to white points (i.e., white or achromatic colors). This indicates the decrease of vividness (saturation) of green. Most plots for soils (shown in red) are distributed around areas corresponding to yellow or orange, with arrows pointing at decreasing values of y (i.e., indicating redder colors), especially for plots in the vicinity of (B). The plot near (C) (shown in blue) corresponds to the spectrum of the open ocean (seawater, Atlantic Ocean), with arrows pointing at increasing values of $y$ (i.e., indicating greener colors).

Figure 11b shows the color palette (as per Fig. 10b) for the obtained $\mathrm{XYZ}$ values corresponding to the 


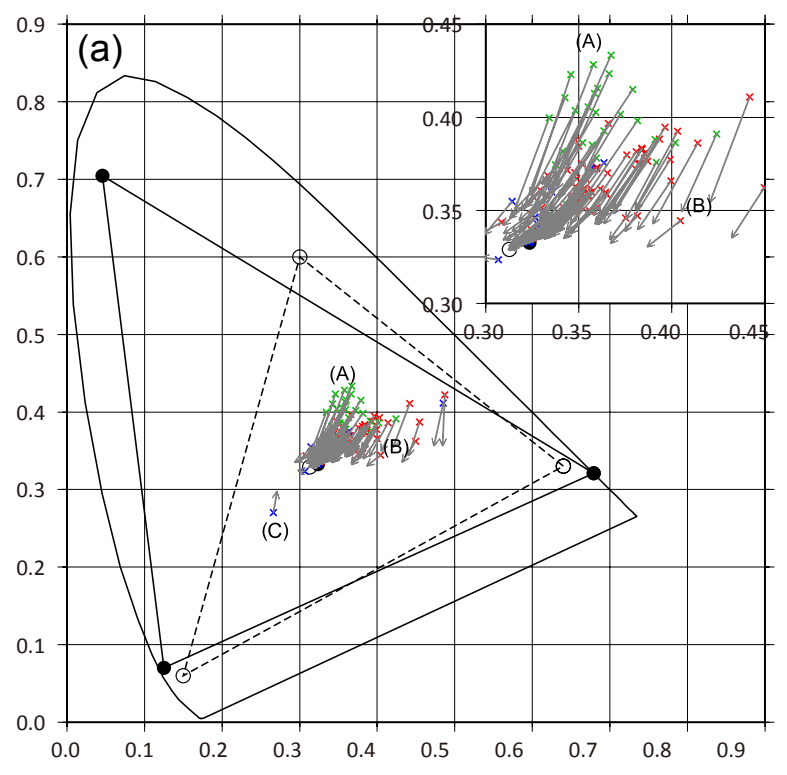

(b)
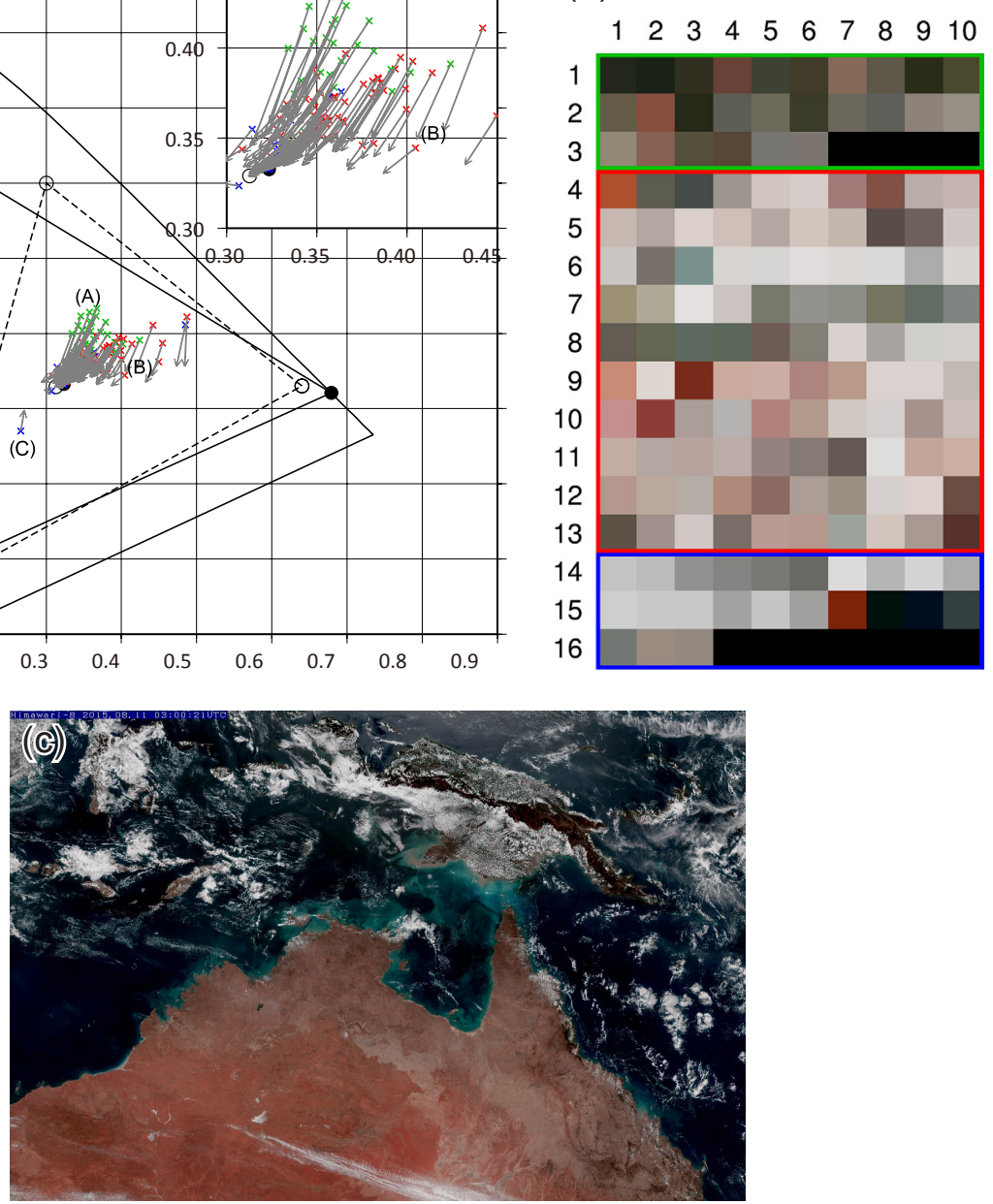

Fig. 11. Evaluation results for imagery from simply composed AHI native RGB bands. (a) Chromaticity diagram indicating the accurate and the obtained chromaticity coordinates. Plot colors are as per Fig. 10a. The color gamuts and white points for AHI and sRGB are as per Fig. 6. (b) Color palette as per Fig. 10b for the obtained XYZ values corresponding to the tips of arrows in (a). (c) AHI imagery around northern Australia at 0300 UTC on 11 August 2015 with Rayleigh scattering correction and log-linear scaling (Section 6) applied. The area and date are as per Fig. 8 of Miller et al. (2016).

arrow tips. Vegetation colors (in the green frame) are less green, and soil colors (in the red frame) are generally redder than the accurate colors shown in Fig. 10b.

Figure 11c shows imagery from the AHI's native RGB band. As noted previously, appearances such as dark tropical vegetation and overly red deserts may be seen based on subjective impression. These characteristics are consistent with the evaluation results shown in Figs. 11a, b and were objectively confirmed using the evaluation technique.

\section{b. Conversion matrix application to AHI native RGB bands}

As discussed previously, the conversion matrix (9) is generated so that the satellite RGB signals can be converted appropriately into XYZ values in consideration of RGB band SRFs. Accordingly, the less accurate coloring of the simple RGB composition shown above is expected to be improved by conversion matrix application. However, the resultant colors appear unnatural (Fig. 7).

XYZ values are obtained by applying matrix (9) to the $\mathrm{AHI}$ native $\mathrm{RGB}$ bands as follows: 

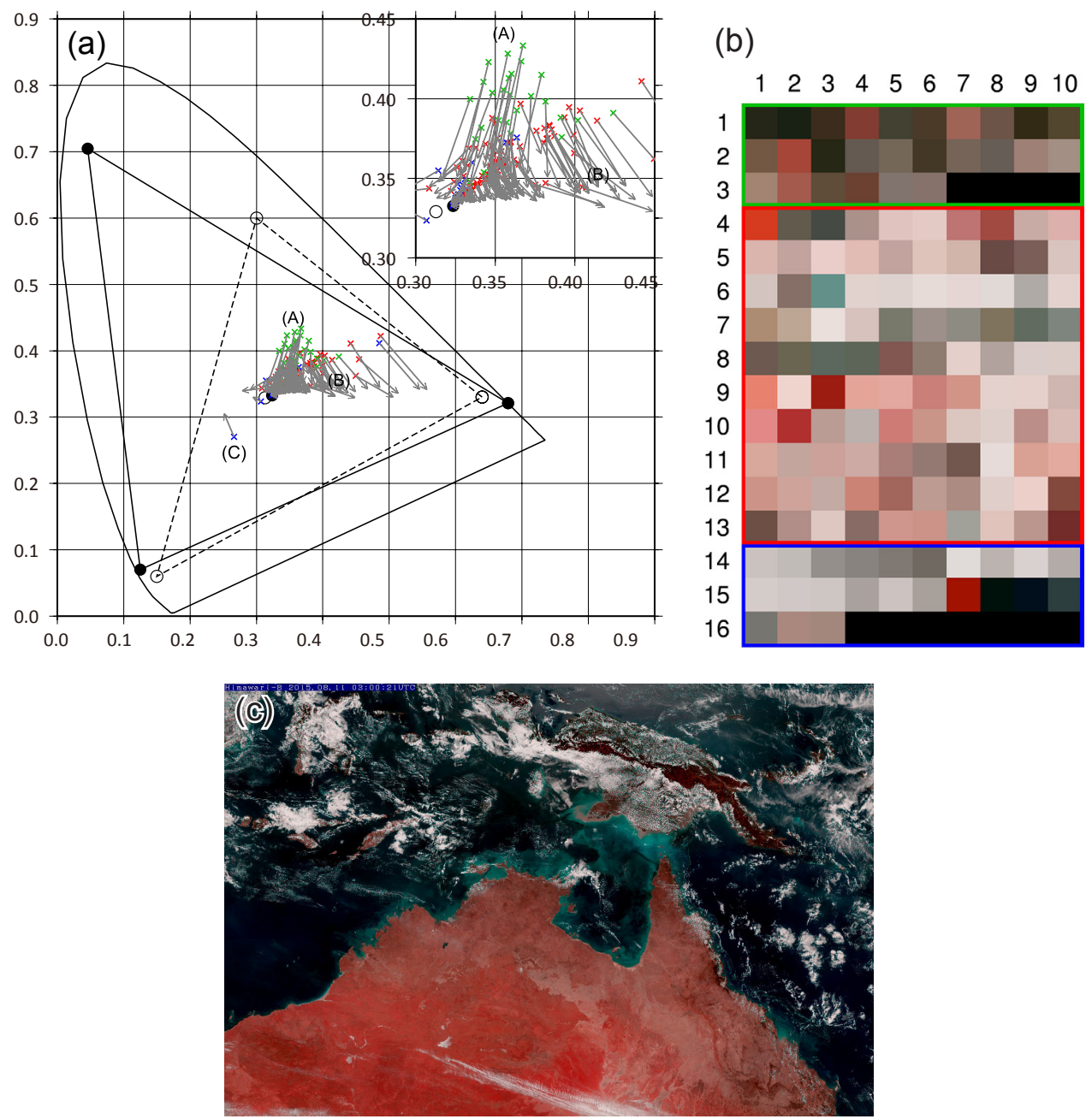

Fig. 12. Evaluation for AHI native RGB bands with a conversion matrix (details as per Fig. 11).

$$
\left(\begin{array}{c}
X \\
Y \\
Z
\end{array}\right)=\left(\begin{array}{ccc}
X_{R_{A H I}} & X_{G_{A H I}} & X_{B_{A H I}} \\
Y_{R_{A H I}} & Y_{G_{A H I}} & Y_{B_{A H I}} \\
Z_{R_{A H I}} & Z_{G_{A H I}} & Z_{B_{A H I}}
\end{array}\right)\left(\begin{array}{l}
R_{A H I} \\
G_{A H I} \\
B_{A H I}
\end{array}\right) .
$$

The resultant chromaticity diagram, color palette, and sample imagery are shown in Fig. 12 as per Fig. 11. As with the unnatural colors in the imagery shown in Fig. 12c, it can be objectively confirmed that the discrepancies from accurate chromaticity coordinates in the chromaticity diagram (Fig. 12a) (i.e., arrow lengths) are larger than those from the simple composition of AHI native bands shown in Fig. 11a. The vegetation plots in the vicinity of (A) still have generally achromatic colors, and the soil plots are more reddish, especially in the vicinity of (B). These characteristics are also seen in the color palette (Fig. $12 b)$.

In summary, appropriate XYZ values cannot be obtained using a combination of AHI native RGB bands even if the conversion matrix is applied. The nature and handling of this issue are discussed in the next section.

\section{Reconstruction of RGB band combination}

As outlined in the previous section, colors were not reproduced appropriately using the combination of AHI native RGB bands. Accordingly, the band combination is discussed in this section.

\subsection{Consideration of the green band}

As mentioned previously, Miller et al. (2016) im- 
proved imagery colors by replacing the native AHI green band with a hybrid green band. Green-band modification was also considered in this study, but the objective was to minimize the discrepancy between the accurate and the obtained $\mathrm{XYZ}$ values by applying a conversion matrix, whereas Miller et al. (2016) focused on boosting the reflectance of vegetation and soil by minimizing the deviation from the VIIRS green band.

For green-band modification, an experiment involving the consideration of a pseudo green band having the same shape of SRF as B02 with various central wavelengths was conducted using the evaluation technique. A conversion matrix was generated for each combination of RGB bands incorporating the pseudo green band, and the overall discrepancies between the accurate and the obtained chromaticity coordinates with wavelength were observed.

The overall discrepancies were expressed as root mean square deviation (RMSD) in a chromaticity diagram where the xy chromaticity coordinates have been converted to $u^{\prime} v^{\prime}$ chromaticity coordinates (CIE 1976 UCS, ISO/CIE 2009), whose distance apart in the diagram is more proportional to the color difference perceived by the human eye.

The relation between $u^{\prime} v^{\prime}$ and xy coordinates is as follows:

$$
\begin{aligned}
& u^{\prime}=\frac{4 x}{-2 x+12 y+3}, \\
& v^{\prime}=\frac{9 y}{-2 x+12 y+3} .
\end{aligned}
$$

Figure 13 shows the RMSD with the amount of central wavelength shift from that of the AHI native green band B02. The RMSD after conversion matrix application is shown as a solid line. It is minimized in case (d), where the shift amount is set to $+45 \mathrm{~nm}$ and the central wavelength is set to around 0.555 $\mu \mathrm{m}$. The RMSD for the simple compositions (i.e., where satellite RGB signals are used as sRGB signals without conversion matrix application) is also shown as a dashed line. A limited improvement was seen, indicating that modification of the green band alone is insufficient to reduce discrepancies and that a conversion matrix is necessary.

Figure 14 shows chromaticity diagrams (as per Fig. 11a) corresponding to cases (a) to (e) in Fig. 13. Case (a) corresponds to the AHI native RGB band combination shown in Fig. 12a. In the minimized case (d), the arrows are generally shorter for most plots, whereas in the other cases, the arrows are longer with system-

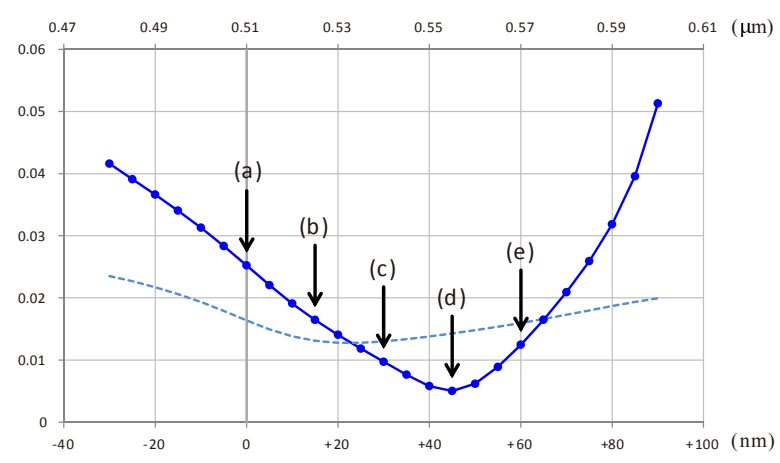

Fig. 13. RMSD of the obtained $u^{\prime} v^{\prime}$ chromaticity coordinates with the amounts of central wavelength shift from that of the native AHI/B02. Solid line: RMSD with conversion matrix application; dashed line: RMSD for simple composition without conversion matrix application (used as sRGB signals). The arrows pointing to the solid line with parenthesized letters correspond to the cases shown in Fig. 14. Bottom horizontal axis: wavelength shift (nm); top horizontal axis: central wavelength $(\mu \mathrm{m})$.

atic directions. The satellite RGB band combination incorporating the shifted green band is assumed to be optimized in case (d).

Below is a discussion of why the native band combination is not optimal with conversion matrix application and why the shifted green band enables discrepancy mitigation. The SRF of the green band (B02) is close to that of the blue band (B01) as shown in Fig. 15 and is not completely independent of B01 with an SRF overlap. Conversely, the central wavelength of the optimal band $(0.555 \mu \mathrm{m}$, the dashed vertical line $)$ is around the middle of the blue (B01) and red (B03) bands and separate from both. This implies that the balance of RGB bands may be an important factor in obtaining $\mathrm{XYZ}$ values. In addition, as reported by Miller et al. (2016), the central wavelength of B02 is somewhat off from the local peak of vegetation reflectance spectra at around $0.55 \mu \mathrm{m}$ (Fig. 15), and the signal received for perceiving vegetation as green may be insufficient.

\subsection{Conversion matrix for optimal RGB band combination}

As discussed above, it was found that XYZ values are appropriately obtained when the pseudo green band is shifted to $0.555 \mu \mathrm{m}$ and the conversion matrix is applied. In consideration of the benefit to be derived in later verification of the pseudo band, we attempted 

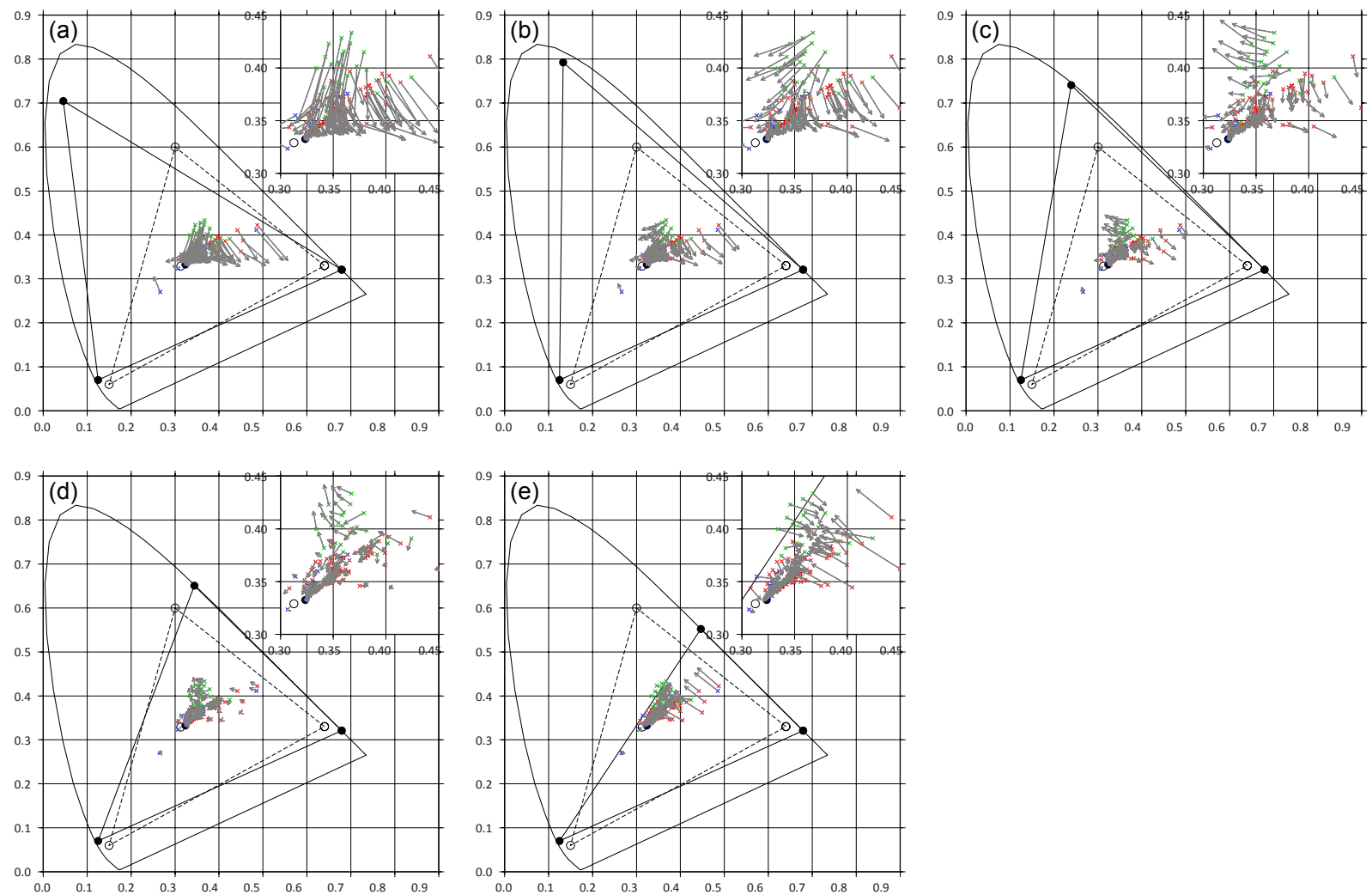

Fig. 14. Chromaticity diagrams based on varied shifting of the B02 SRF with conversion matrix application (corresponding to the cases shown in Fig. 13). (a) Native AHI B02 (central wavelength $0.510 \mu \mathrm{m}$ ), (b) shifted $+15 \mathrm{~nm}(0.525 \mu \mathrm{m})$, (c) shifted $+30 \mathrm{~nm}(0.540 \mu \mathrm{m})$, (d) shifted $+45 \mathrm{~nm}(0.555 \mu \mathrm{m})$, and (e) shifted $+60 \mathrm{~nm}(0.570$ $\mu \mathrm{m})$. Solid triangles: color gamut of satellite sensors incorporating the shifted green band. Dashed triangles: color gamut of sRGB.

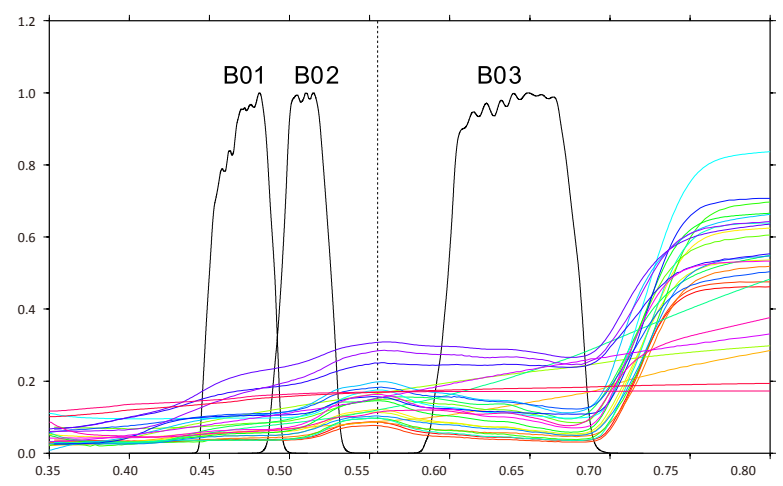

Fig. 15. SRFs of the AHI visible band, reflectance spectra of the vegetation shown in Fig. 9a, and optimal central wavelength $(0.555 \mu \mathrm{m})$ (vertical line). to find an SRF of an existing sensor that provides a level of performance comparable to that of the shifted green band. Application of the evaluation technique identified MODIS/B04 aboard the Aqua satellite as such a band (this was closer than other candidate bands such as VIIRS/M04), and this was subsequently adopted as the SRF for the pseudo green band.

The conversion matrix for the new combination incorporating the pseudo green band (i.e., MODIS/B04) is generated as described in Section 3.1. The SRF was obtained from the MODIS Characterization Support Team webpage (http://mcst.gsfc.nasa.gov/calibration/ parameters). The chromaticity coordinates of MODIS/ B04 necessary for the conversion matrix are obtained using Eqs. (6) and (7), with Table 3 replacing Table 5. The conversion matrix (9) is replaced with the following matrix (20), which is obtained by applying Table 5 to Eqs. (4) and (5): 
Table 5. Chromaticity coordinates of the new RGB bands (replacement of AHI/ B02 with MODIS/B04 in Table 3).

\begin{tabular}{ccccc}
\hline $\begin{array}{c}\text { Chromaticity } \\
\text { coordinates }\end{array}$ & $\begin{array}{c}\text { Red } \\
(\mathrm{AHI} / \mathrm{B} 03)\end{array}$ & $\begin{array}{c}\text { Green } \\
\text { (MODIS/B04) }\end{array}$ & $\begin{array}{c}\text { Blue } \\
(\mathrm{AHI} / \mathrm{B} 01)\end{array}$ & $\begin{array}{c}\text { White } \\
\text { (Sunlight) }\end{array}$ \\
\hline$x$ & 0.6789 & 0.3320 & 0.1252 & 0.3236 \\
$y$ & 0.3209 & 0.6632 & 0.0702 & 0.3326 \\
$z=1-x-y$ & 0.0002 & 0.0048 & 0.8046 & 0.3438 \\
\hline
\end{tabular}

$$
\left(\begin{array}{ccc}
X_{R_{A H I}} & X_{G_{M O D I S}} & X_{B_{A H I}} \\
Y_{R_{A H I}} & Y_{G_{\text {MODIS }}} & Y_{B_{A H I}} \\
Z_{R_{A H I}} & Z_{G_{\text {MODIS }}} & Z_{B_{A H I}}
\end{array}\right)=\left(\begin{array}{lll}
0.4677 & 0.3450 & 0.1600 \\
0.2210 & 0.6892 & 0.0897 \\
0.0001 & 0.0050 & 1.0285
\end{array}\right) .
$$

The remapping matrix in Eq. (12) is replaced with the following, and this is subsequently used to render TCR imagery (Section 6):

$$
\begin{aligned}
& \left(\begin{array}{l}
R_{s R G B} \\
G_{s R G B} \\
B_{s R G B}
\end{array}\right)=\left(\begin{array}{ccc}
X_{R_{s R G B}} & X_{G_{s R G B}} & X_{B_{s R G B}} \\
Y_{R_{S R G B}} & Y_{G_{S R G B}} & Y_{B_{s R G B}} \\
Z_{R_{s R G B}} & Z_{G_{s R G B}} & Z_{B_{s R G B}}
\end{array}\right)^{-1}\left(\begin{array}{c}
X \\
Y \\
Z
\end{array}\right) \\
& =\left(\begin{array}{ccc}
X_{R_{S R G B}} & X_{G_{S R C B}} & X_{B_{S R C B}} \\
Y_{R_{S R C B}} & Y_{G_{S R G B}} & Y_{B_{S R C B}} \\
Z_{R_{S R C B}} & Z_{G_{S R C B}} & Z_{B_{S R C B}}
\end{array}\right)^{-1} \\
& \times\left(\begin{array}{ccc}
X_{R_{A H I}} & X_{G_{\text {MODIS }}} & X_{B_{A H I}} \\
Y_{R_{A H I}} & Y_{G_{M O D I S}} & Y_{B_{A H I}} \\
Z_{R_{A H I}} & Z_{G_{\text {MODIS }}} & Z_{B_{A H I}}
\end{array}\right)\left(\begin{array}{c}
R_{A H I} \\
G_{M O D I S} \\
B_{A H I}
\end{array}\right) \\
& =\left(\begin{array}{rrr}
1.1759 & 0.0561 & -0.1322 \\
-0.0386 & 0.9587 & 0.0559 \\
-0.0189 & -0.1161 & 1.0777
\end{array}\right)\left(\begin{array}{c}
R_{A H I} \\
G_{M O D I S} \\
B_{A H I}
\end{array}\right) \text {. }
\end{aligned}
$$

\subsection{Generation of pseudo green band data}

Observation data from the pseudo green band (i.e., MODIS/B04) need to be generated, as no such band is actually present on Himawari-8. Like in the research by Miller et al. (2016), which involved the generation of a hybrid green band from B02 and B04 with blend factor $\mathrm{F}$, the pseudo green band was generated from existing bands. This study adopted multiple regression analysis with existing bands as predictor variables for the generation of the pseudo green band. The simulated reflectance dataset generated from the reflectance spectra library described in Section 4 was applied for regression.

As shown in Table 1, there are six candidate reflection bands within the visible to near-infrared wave- length range. Besides the native green band $\mathrm{B} 02, \mathrm{~B} 04$ $(0.86 \mu \mathrm{m})$ is a significant band for regression, where reflectance is higher for land surfaces such as vegetation and soil (Fig. 9), which was also utilized for the hybrid green band. B05 $(1.6 \mu \mathrm{m})$ and B06 $(2.3 \mu \mathrm{m})$ were not considered for use because of their higher sensitivity to cloud particle phase and size, which were not taken into account in this study. Intervariable correlations were also taken into account, and eventually B02, B03, and B04 were chosen as predictor variables. It is possible to choose two bands as predictor variables (B02 and B04), but the regression error is larger than the tree-band regression.

The regression coefficients are derived by forcing the intercept to 0 so that the pseudo band value is 0 when all predictor bands are 0 (i.e., no illumination). The coefficients are also adjusted such that their sum is 1 so that the pseudo band value is also 1 when all predictor variables are 1 (i.e., white material).

With regression, the pseudo green band is derived as follows:

$$
\begin{aligned}
G_{M O D I S}= & 0.6321 \times G_{A H I}+0.2928 \times R_{A H I} \\
& +0.0751 \times N_{A H I},
\end{aligned}
$$

where $G_{M O D I S}, G_{A H I}, R_{A H I}$, and $N_{A H I}$ are MODIS/B04, AHI/B02, AHI/B03, and AHI/B04, respectively.

Equation (22) was confirmed via application of the predictor bands and compared with the target band. Figure 16a shows scatter plot results from the comparison of the simulated reflectance dataset used for regression analysis. A good agreement with most plots distributed around the diagonal line was observed. Figure 16b shows two-dimensional histogram results from comparison using collocated AHI data (after Rayleigh scattering correction) and MODIS data (atmospheric corrected dataset MYD09; NASA 2015) for a certain scene. Although a large deviation was observed due to rough collocation without consideration of the satellite zenith angle and parallax, distribution was generally near the diagonal line and no significant bias was observed. For further improvement, a detailed investigation of these differences is required. 
(a)

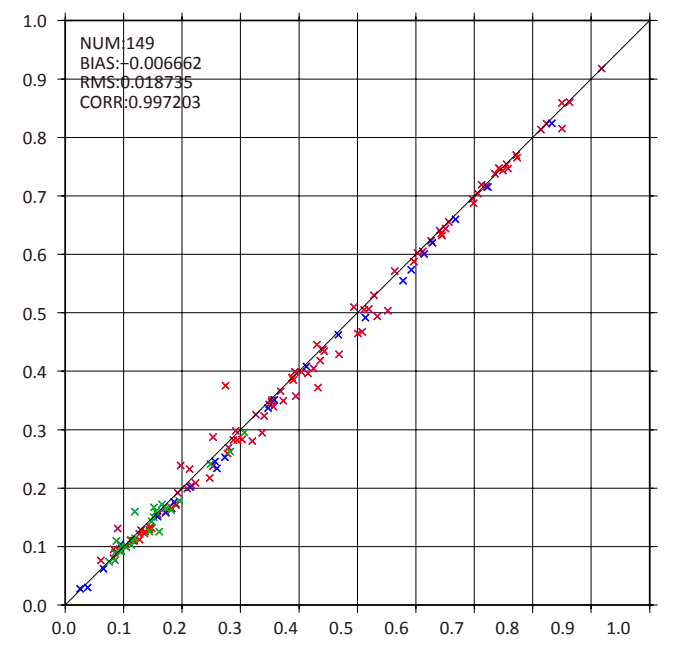

(b)

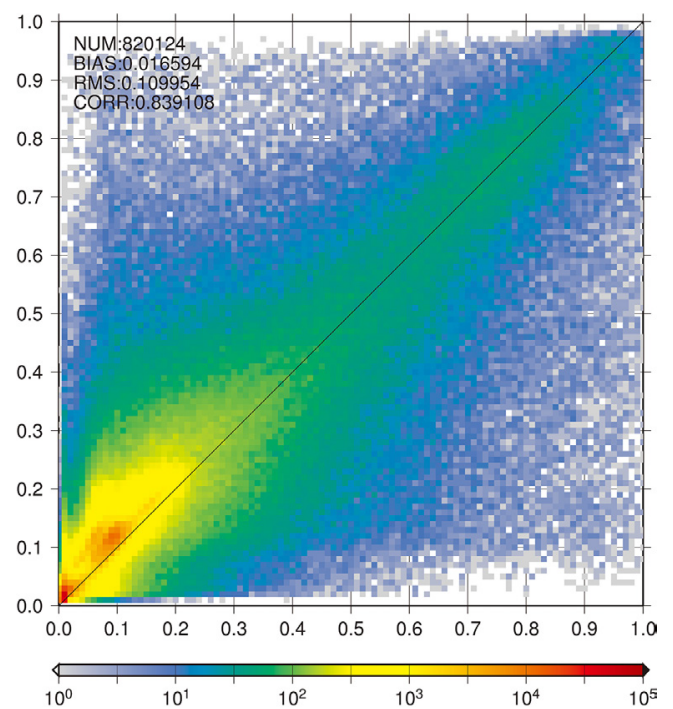

Fig. 16. Checking of the regression formula (22) for the pseudo green band. Horizontal axis: MODIS/B04; vertical axis: regressed MODIS/B04 from AHI data. (a) Scatter plots based on the simulated reflectance dataset. Plot colors are as per Fig. 10a. (b) Two-dimensional histogram from the collocated AHI and MODIS dataset for a certain scene (multiple granules over eastern China and northern and eastern Australia including land and sea, 8 August 2015, not shown).
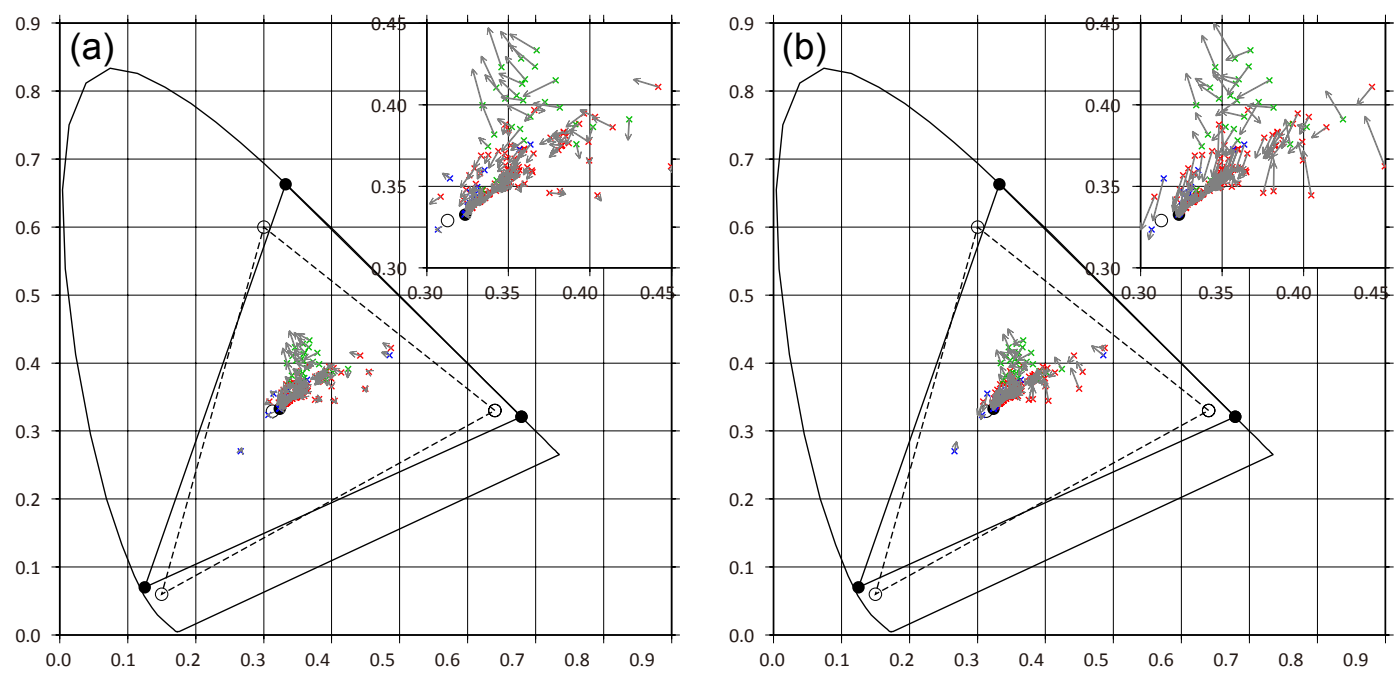

Fig. 17. Chromaticity coordinates after conversion matrix application. (a) With simulated MODIS/B04 data (i.e., perfect regression). (b) With regressed MODIS/B04 data from the regression formula (22) with existing bands.

Regression error sensitivity to XYZ values was checked in chromaticity diagrams. Figures $17 \mathrm{a}, \mathrm{b}$ show the differences in the accurate and the obtained chromaticity coordinates after conversion matrix application. Figure $17 \mathrm{a}$ shows the results of the application of simulated MODIS/B04 data as the green band (i.e., perfect regression), and Fig. 17b shows the results of the replacement of the green band with regressed data generated using Eq. (22). Although the deviations in Fig. $17 \mathrm{~b}$ are slightly larger than those in Fig. 17a, 
(a)

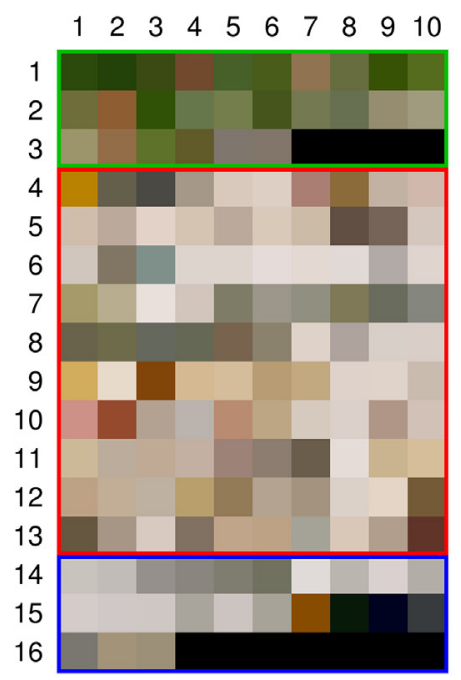

(b)

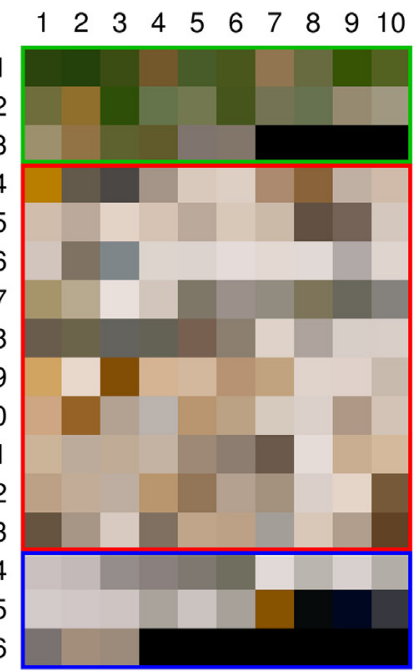

(c)

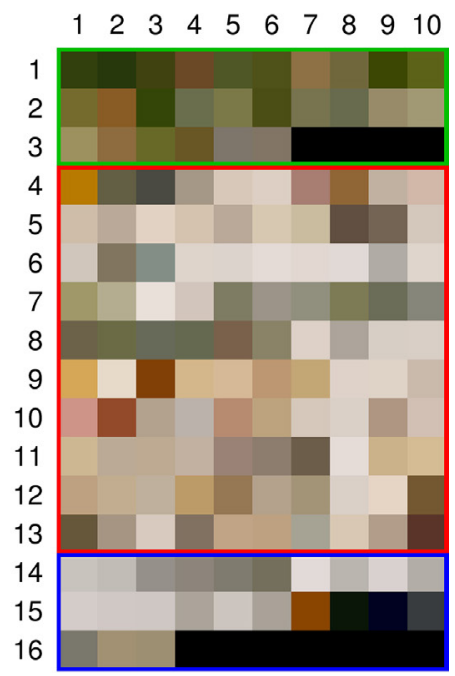

Fig. 18. Color palettes of interpreted colors from XYZ values. (a) Colors reproduced using simulated MODIS/ B04 data (i.e., perfect regression). (b) Colors reproduced using regressed MODIS/B04 data with the regression formula (22) for existing bands. (c) Accurate colors.

there are no significant deviations as a whole and the results are considered appropriate.

Figure 18 shows color palettes with the colors in sRGB display interpreted from XYZ values. The color palettes (Figs. 18a, b) correspond to the obtained chromaticity coordinates shown in Figs. 17a, b respectively, and Fig. 18c shows accurate colors (shown again, as per Fig. 10b). Although slight differences were observed, all color palettes are similar and the resultant colors shown in Fig. 18b closely match the accurate ones shown in Fig. 18c.

Figure 19 shows AHI imagery rendered with the pseudo green band generated using Eq. (22) and the remapping matrix (21) (i.e., TCR imagery). The colors are more realistic than those produced via simple composition of the AHI native band (Fig. 11c). This imagery is considered to reproduce colors as accurately as possible with current best efforts.

\section{True Color Reproduction (TCR) imagery}

The new true color imagery based on the color reproduction approach outlined in this article was termed True Color Reproduction (TCR) imagery. Atmospheric (Rayleigh scattering) correction and log-linear scaling (Miller et al. 2016) are also applied using methods provided by CIRA to the imagery. A flowchart of imagery rendering is shown in Fig. 20, and the process is outlined below.

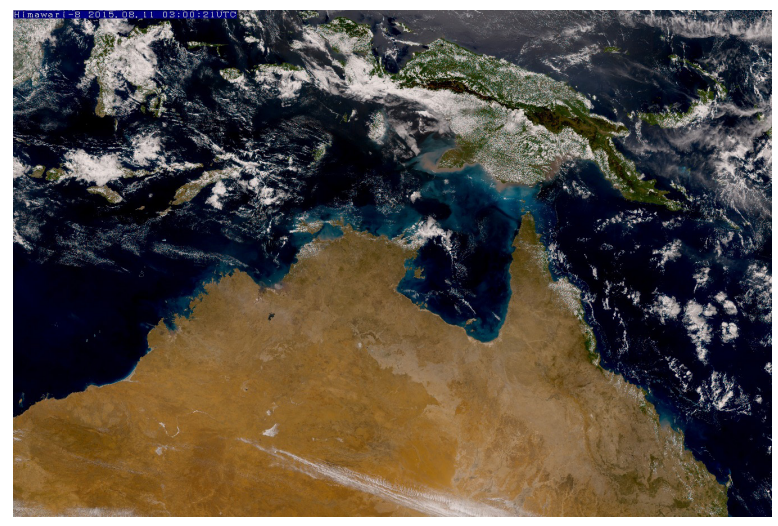

Fig. 19. AHI imagery with the regressed pseudo green band and the remapping matrix. The area and date are as per Fig. 11c.

(i) Rayleigh scattering contribution from the atmosphere to reflectance is removed from the source imagery (i.e., B01, B02, B03, and B04) using the CIRA program, with B13 $(10.4 \mu \mathrm{m})$ data taken as a proxy of cloud height to allow adjustment of the scattering. This process makes imagery as clear as if viewed in proximity to the surface and facilitates the visualization of thin haze and aerosol clouds. 


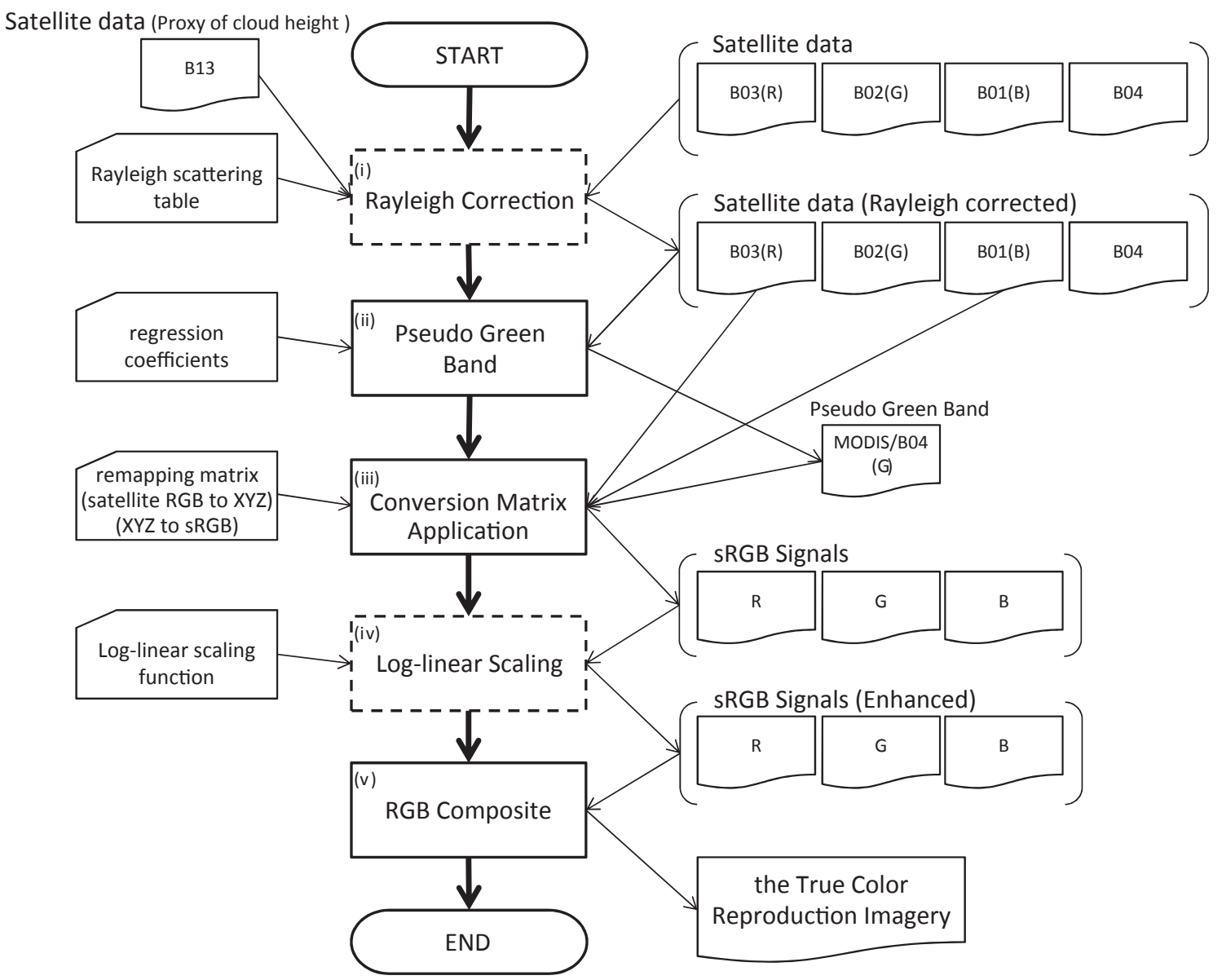

Fig. 20. TCR imagery flowchart. The procedures indicated with dashed-line boxes are provided courtesy of CIRA.

(ii) The pseudo green band with a central wavelength of around $0.555 \mu \mathrm{m}$ (i.e., MODIS/B04) is generated by applying the regression formula (22).

(iii) The remapping matrix in Eq. (21) is applied to the above satellite RGB data for conversion to RGB signals of the sRGB standard.

(iv) Using the CIRA program, log-linear scaling is applied to RGB signals to mimic the nonlinear response of human vision. Imagery is generally brightened (i.e., enhanced) as a result.

(v) TCR imagery is generated via composition of enhanced RGB signals.

Processing with a local computer system (equipped with $256 \mathrm{~GB}$ of RAM and 12-core Intel Xeon CPU E5-2690) takes around $80 \mathrm{~s}$ for full-disk imagery (Fig. 1b) and around $4 \mathrm{~s}$ for regional imagery (Fig. 19). Although processing can take up to $60 \%$ longer than with simple RGB composition imagery because of the additional processes involved (especially for Rayleigh scattering correction), the increase is considered to be of limited significance and therefore acceptable.

This imagery was added to the JMA/MSC website in May 2016 and used to support the first images from Himawari-9 in January 2017 (Japan Meteorological Agency 2017).

\section{Discussion}

\subsection{True color imagery comparison}

Figure 21 shows AHI true color imagery variations of the previous scene covering around northern Australia. Rayleigh scattering correction and log-linear scaling (see Section 6) are applied in all cases. Figure 21a shows a simple composition of the AHI native band combination (as shown Fig. 11c). Figure 21b shows an imagery rendered with the "hybrid, atmospherically corrected (HAC)" approach (Miller et al. 2016), in which the AHI green band is replaced with a hybrid green band expressed in the following formula 


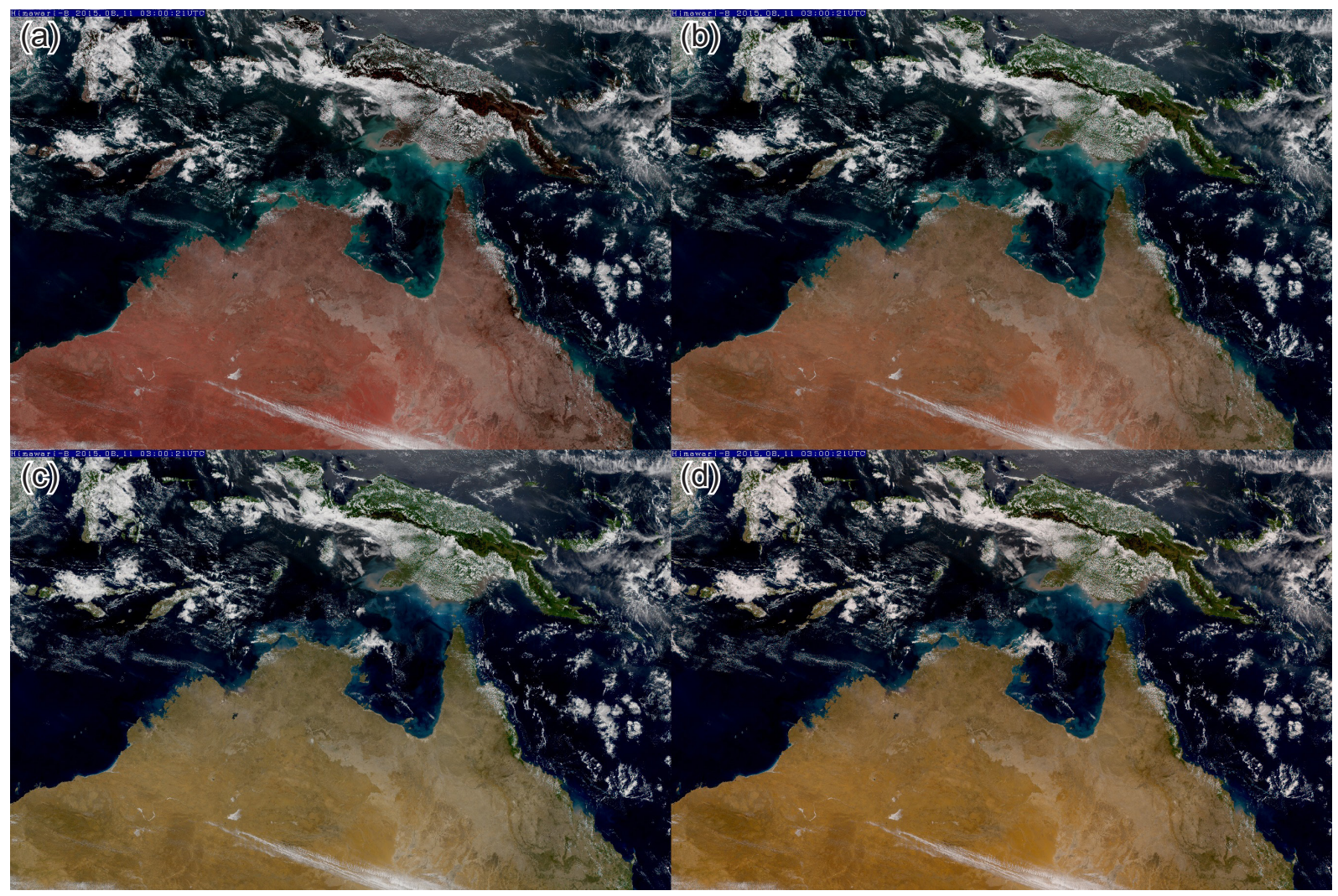

Fig. 21. AHI true color imagery of northern Australia for 0300 UTC on 11 August 2015. (a) Simple composition of the AHI native RGB band combination. (b) HAC approach (Miller et al. 2016). (c) Green replaced with pseudo green without remapping matrix application. (d) TCR imagery.

with a blend factor of $F=0.07$ :

\section{Hybrid green}

$$
=(1-F) \times R(0.51 \mu \mathrm{m})+F \times R(0.86 \mu \mathrm{m}) .
$$

Figure 21c shows a composition of the new combination of AHI RGB bands for TCR imagery in which the green band is replaced with a pseudo green band generated using Eq. (22) but remapping matrix application is omitted. This imagery is intended to highlight differences before and after remapping matrix application rather than being for actual usage. Figure $21 \mathrm{~d}$ shows TCR imagery rendered by applying the remapping matrix in (21) to the imagery in Fig. 21c (duplicated in Fig. 19).

Color differences are observed among all imageries. Figures $21 \mathrm{~b}-\mathrm{d}$ appear realistic based on subjective impression. Soils (e.g., over the Australian Outback) appear overly red in Fig. 21a, but this is mitigated in Figs. 21b-d. Figure 21c appears somewhat greenish or less vivid, and yellowish or orangish tones are seen in Fig. 21d. Vegetation (e.g., over New Guinea Island) is unnaturally dark brown or gray in Fig. 21a. The green parts in Figs. 21b-d appear fine, and comparison shows no significant differences among them in this scene.

Figure 22 shows chromaticity diagrams corresponding to the imagery in Figs. 21a-d to consider the color differences in detail as per the diagrams shown already (e.g., Fig. 11a). For Figs. 22a-c, XYZ values corresponding to the arrow tips are obtained by applying the sRGB conversion matrix (10) to RGB signals under the assumption that the output device is compliant with the sRGB standard. For Fig. 22d, XYZ values are obtained by applying the matrix (20) for the new RGB band combination incorporating the pseudo green band.

Figure 23 shows color palettes interpreting the XYZ values at the arrow tips in the corresponding chromaticity diagrams of Fig. 22. The color palette (Fig. 23e) 

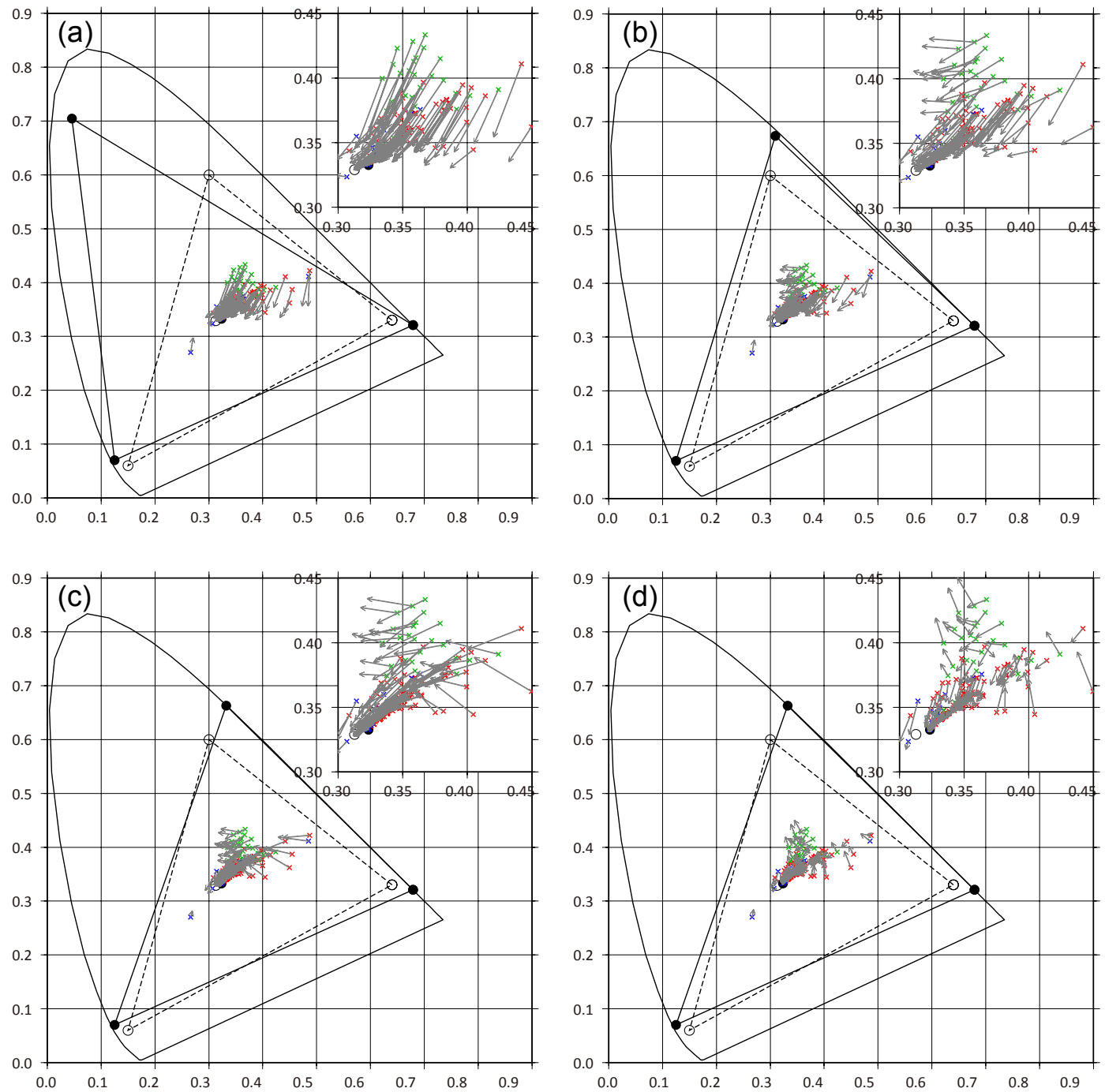

Fig. 22. Chromaticity diagrams corresponding to Figs. 21a-d. The details are as per Fig. 11a. Note: The chromaticity coordinates of the green band (the upper apex of the solid triangle) in (b) indicate Suomi-NPP/VIIRS/M4 $(0.555 \mu \mathrm{m})$, which is used for determination of the hybrid green blending factor $\mathrm{F}$ in the HAC approach.

shows accurate colors (also shown in Fig. 10b).

As discussed already in Section 4.2a, the unnatural colors for vegetation and soil seen in the simple composition imagery of Fig. 21a are observed consistently in the chromaticity diagram of Fig. 22a and the color palette of Fig. 23a. Improved colors seen in imagery produced using the HAC approach (Fig. 21b) are observed in related chromaticity diagrams (Fig. 22) and color palettes (Fig. 23). The arrows in Fig. 22b are generally shorter than those of Fig. 22a, which implies general color improvement. With regard to vegetation plots (shown in green), the arrow tips in Fig. 22a are near the white point (representing achromatic colors) but closer to the accurate values in Fig. 22b. This indicates color improvement with a change from gray to green (see also Fig. 3, which demonstrates the approximate relationship between coordinates and colors). A color difference is also seen with the color palettes in Fig. 23. The vegetation colors in the green frame of Fig. 23a appear generally achromatic, whereas those in Fig. 23b appear greener. However, the arrow tips in Fig. 22b are still slightly shifted to the left from the accurate plots, meaning that the hues of the reproduced green colors differ slightly from the 

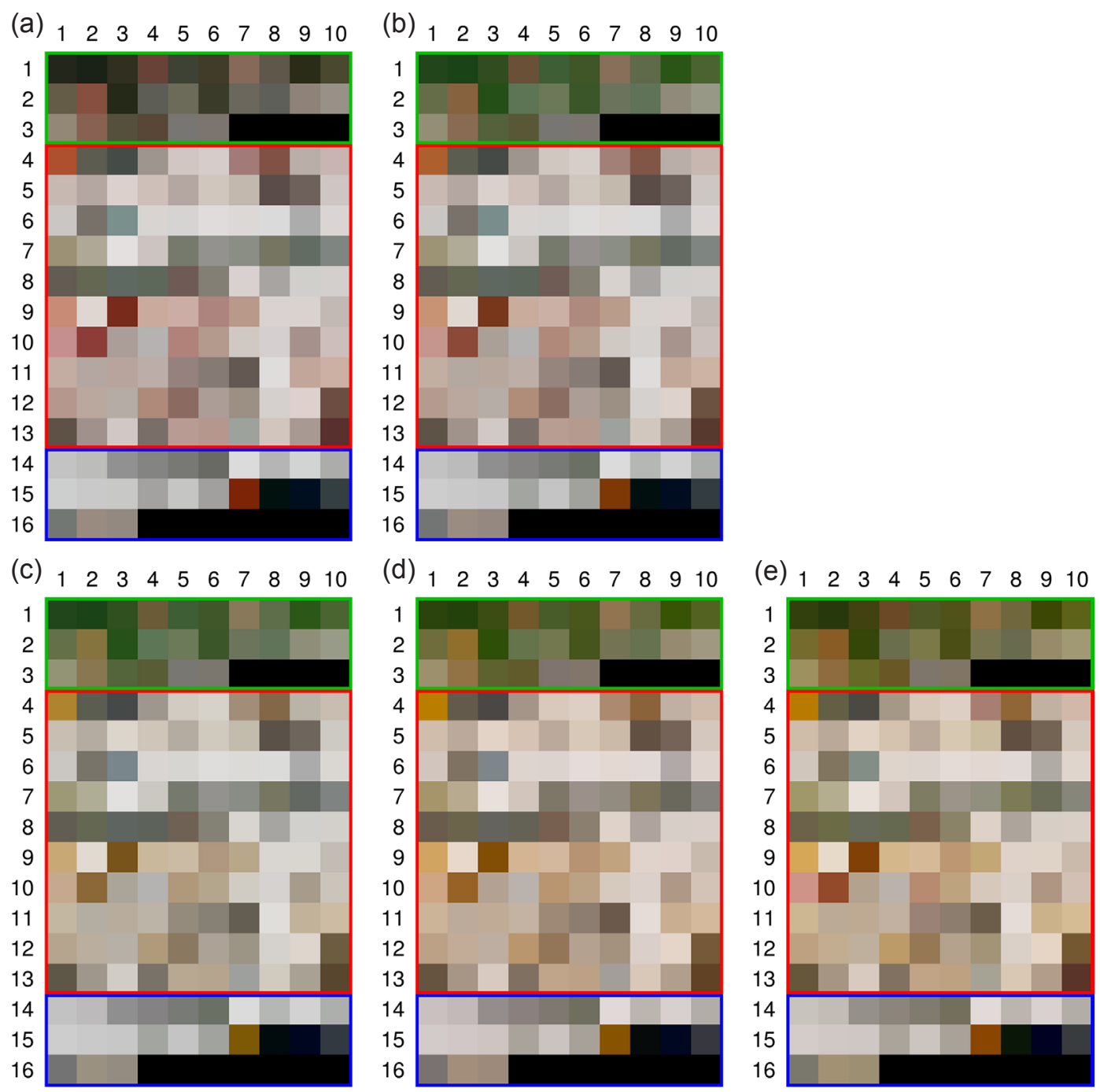

Fig. 23. Color palettes corresponding to the XYZ values in Figs. 22a-d. (e) color palette for accurate colors. The details are as per Fig. 11b.

accurate colors. This can be seen by comparing the color palettes in Figs. 23b, e. The vegetation color difference is also discussed later in Section 7.2b. With regard to the soil plots (shown in red) in Fig. 22b, the $y$ coordinates at the arrow tips are generally greater than those of Fig. 22a, indicating the mitigation of overly red colors. However, the coordinates are still shifted to the lower left of the accurate plots, and the resultant colors are generally reddish as shown in the red frame of the color palette of Fig. 23b compared with the accurate colors in Fig. 23e. The general differences in color between Figs. 23a and 23b appear consistent with those in Figs. 21a, b.
The difference between Figs. 21c and 21d shows the effect of the color conversion via XYZ values discussed in this study. With matrix application, the arrow lengths become shorter as found in the diagram of Fig. 22d, and the colors interpreted from the XYZ values shown in Fig. $23 \mathrm{~d}$ become very similar to the accurate colors in Fig. 23e. With regard to vegetation plots (shown in green), the arrow tips in the chromaticity diagram Fig. 22c are slightly shifted to the left from the accurate plots similar to those in Fig. 22b described above.

With regard to the plots of soils (shown in red), the arrow tips in the chromaticity diagram (Fig. 22c) 
are closer to the white point than in Fig. 22d, and the interpreted colors shown in the red frame of the color palette in Fig. 23c generally appear less vivid than in Fig. 23d. The general differences in color between Figs. $23 \mathrm{c}$ and $23 \mathrm{~d}$ are also consistent with the difference of the imagery in Figs. 21c, d. Accordingly, the colors in Fig. 21d are considered similar to the accurate colors.

The discussion of the differences between Figs. 21b and $21 \mathrm{~d}$ is similar to the above. The arrow tips (Figs. $22 \mathrm{~b}, \mathrm{~d}$ ) and colors interpreted from XYZ values (Figs. 23b, d) infer that soil colors in Fig. 21b deviate from accurate colors and appear reddish compared with those in Fig. 21d. These differences are consistently found in actual imagery of the Australian Outback.

In summary, the color differences in the true color imagery of Fig. 21 have been objectively discussed here on the basis of the obtained XYZ values shown as chromaticity diagrams in Fig. 22 and color palettes in Fig. 23.

\subsection{Case studies}

Cases illustrating the utility of TCR imagery are discussed below to highlight its effectiveness.

\section{a. Dust storm}

Figure 24 shows a scene from northern Japan's Hokkaido area for 8 May 2016. A haze (or smoke) cloud can be seen approaching from the west over the Sea of Japan. A dust cloud stirred up by strong winds blowing over dry fields in southeastern Hokkaido also blows in an east-southeasterly direction. Owing to

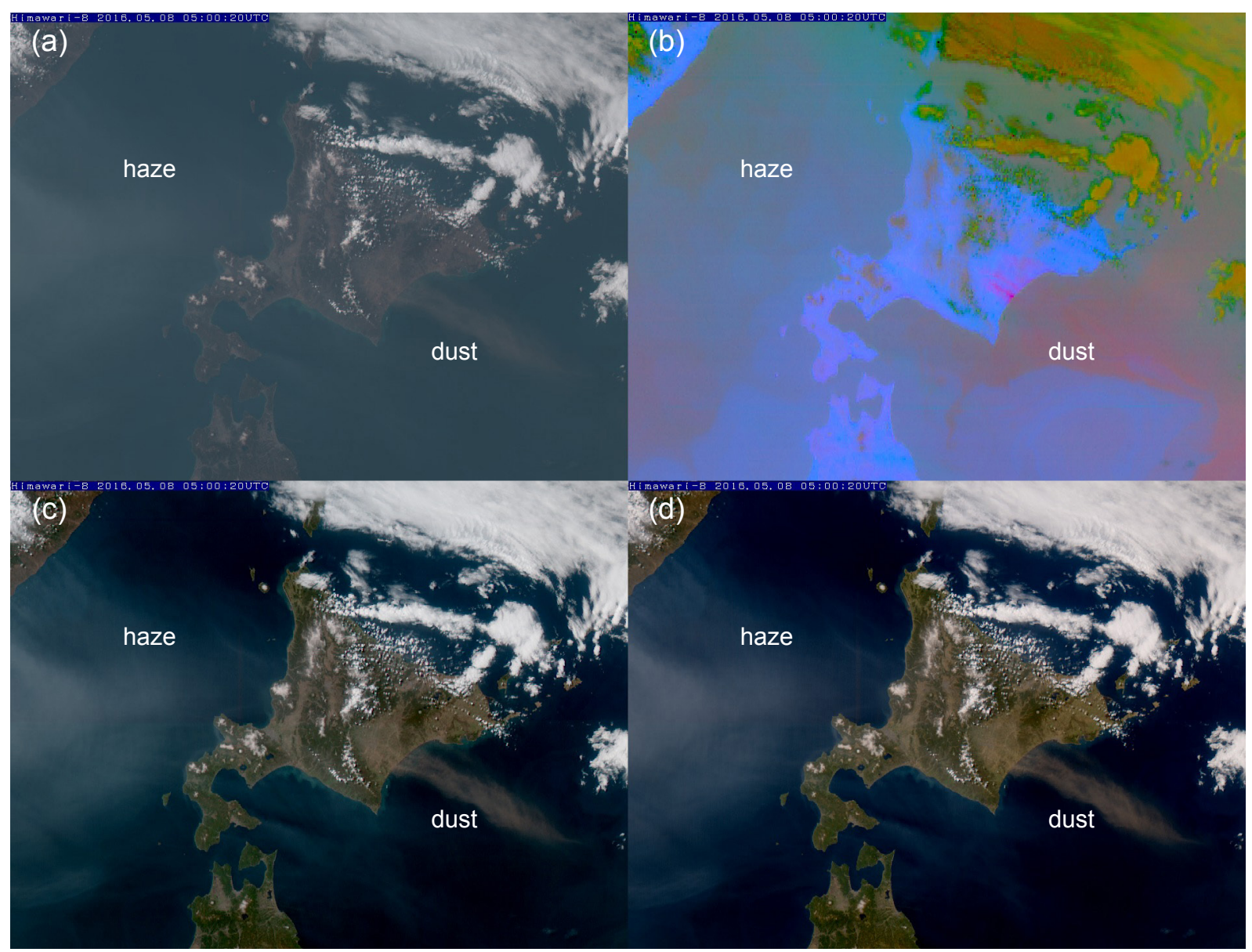

Fig. 24. True color imagery northern Japan's Hokkaido area (8 May 2016, 0500 UTC). (a) Simple composition of AHI native RGB bands (enhanced with gamma correction, $\Gamma=2.0$ ). (b) Dust RGB imagery (color composite for dust identification consisting of red: B15 $(12.4 \mu \mathrm{m})-\mathrm{B} 13(10.4 \mu \mathrm{m})$; green: B13 - B11 $(8.6 \mu \mathrm{m})$; and blue: B13). (c) Imagery based on the HAC approach (Miller et al. 2016). (d) TCR imagery. 
Rayleigh scattering correction, both phenomena are clearer in the HAC imagery (Fig. 24c) and TCR imagery (Fig. 24d) than in the simple true color imagery (Fig. 24a). The dust cloud is likely to be soil-colored, making it easy to identify in Figs. 24c, d over sea. However, it is unclear over land as the color is very similar to the background.

Figure 24b shows a Dust RGB enhancement, which highlights mineral particles such as Asian dust (yellow sand) and volcanic ash in a pinkish color. There is a pinkish area corresponding to the dust cloud over land, but it is unclear over the sea. The Dust RGB imagery and true color imagery (i.e., TCR and HAC) exhibit a complementary relationship for the detection of dust cloud in this case. Although there are slight differences in color as discussed in Section 7.1, the true color imageries in Figs. 24c, d both appear realistic based on subjective impression.

\section{b. Seasonal changes in vegetation over central Japan}

Figure 25 shows true color imagery of central Japan for 15 October 2016, 13 November 2016, 16 February 2017, and 20 May 2017. The panels in Fig. 25a show a simple composition imagery from AHI native RGB bands, and those in Figs. 25b, c show HAC and TCR imagery, respectively. Rayleigh scattering correction and log-linear scaling (see Section 6) are applied for all images.

It can be seen that vegetation in the simple composition imagery of Fig. 25a is generally not expressed as green, appearing dark brown or gray over mountainous areas considered to be covered with greenery. The HAC (Fig. 25b) and TCR (Fig. 25c) imageries effectively express green colors and generally appear realistic. As the season progresses from autumn to spring (from top to bottom in the figure), a clear change in vegetation color from green to brown (autumn leaves) and from brown (and snow) to green again is observed in Figs. 25b, c for the mountainous areas, whereas an unnatural dark red is found for the autumn leaves in Fig. 25a.

There are slight color differences between Figs. $25 \mathrm{~b}$, c. The green of vegetation is slightly deeper in Fig. 25b and slightly light or yellowish in Fig. 25c. This is also seen in the relevant chromaticity diagrams (Figs. 22b, d) and color palettes (Figs. 23b, d) as discussed in Section 7.1. The chromaticity coordinates of vegetation plots are farther to the right in the HAC imagery (Fig. 22b) than in the TCR imagery (Fig. 22d), and the resultant colors in the color palettes show similar differences between actual images.

\subsection{Application for the other satellites}

The color reproduction approach discussed in this study is also applicable to other satellites that have several bands within the visible to near-infrared wavelength range. The steps involved are described below.

(i) The accuracy of simple true color imageries based on a composition of native RGB bands is verified using the evaluation technique discussed in Section 4.2a. XYZ values are obtained by applying the conversion matrix for the outpour device (e.g. matrix (10) for the sRGB standard). If the deviation from accurate values is assumed to be small, the RGB band data can be used as RGB signals as is with no need for consideration of differences in RGB primary colors between the satellite and the output device. However, this case might be rare.

(ii) To account for RGB primary color differences, the conversion matrix for the combination of satellite RGB bands discussed in Section 3.1 is applied to the satellite RGB signals. If discrepancies between the obtained XYZ values and the accurate values are sufficiently small, the RGB signals for the output device can be obtained by converting the $\mathrm{XYZ}$ values.

(iii) If conversion matrix application fails to reduce discrepancies, the replacement of an RGB band with an alternative may be considered.

(iv) If there are no alternative bands or application of an alternative fails to reduce discrepancies, a pseudo band is considered to be generated from other existing bands. The procedure for determining the optimal pseudo band is discussed in this study for AHI. This case is relevant to most newgeneration geostationary satellites with no green band (e.g., GOES-16/ABI, FY-4A/AGRI) and those with a green band around $0.51 \mu \mathrm{m}$ (e.g., MTG/FCI, GEO-KOMPSAT-2A/AMI).

For verification of this approach, color consistency among different sensors needs to be checked using actual imagery as a next step. For this work, other issues also need to be considered such as the dependence of the reflectance spectra on the solar/satellite direction angles, collocation error, parallax, and calibration bias.

\subsection{Other considerations}

a. Limitations depending on reflectance spectra

Small residuals between the obtained and the accurate XYZ values (or chromaticity) remain, as shown in Fig. 17, even for the perfect regression. One of the possible causes is the limited spectral coverage 


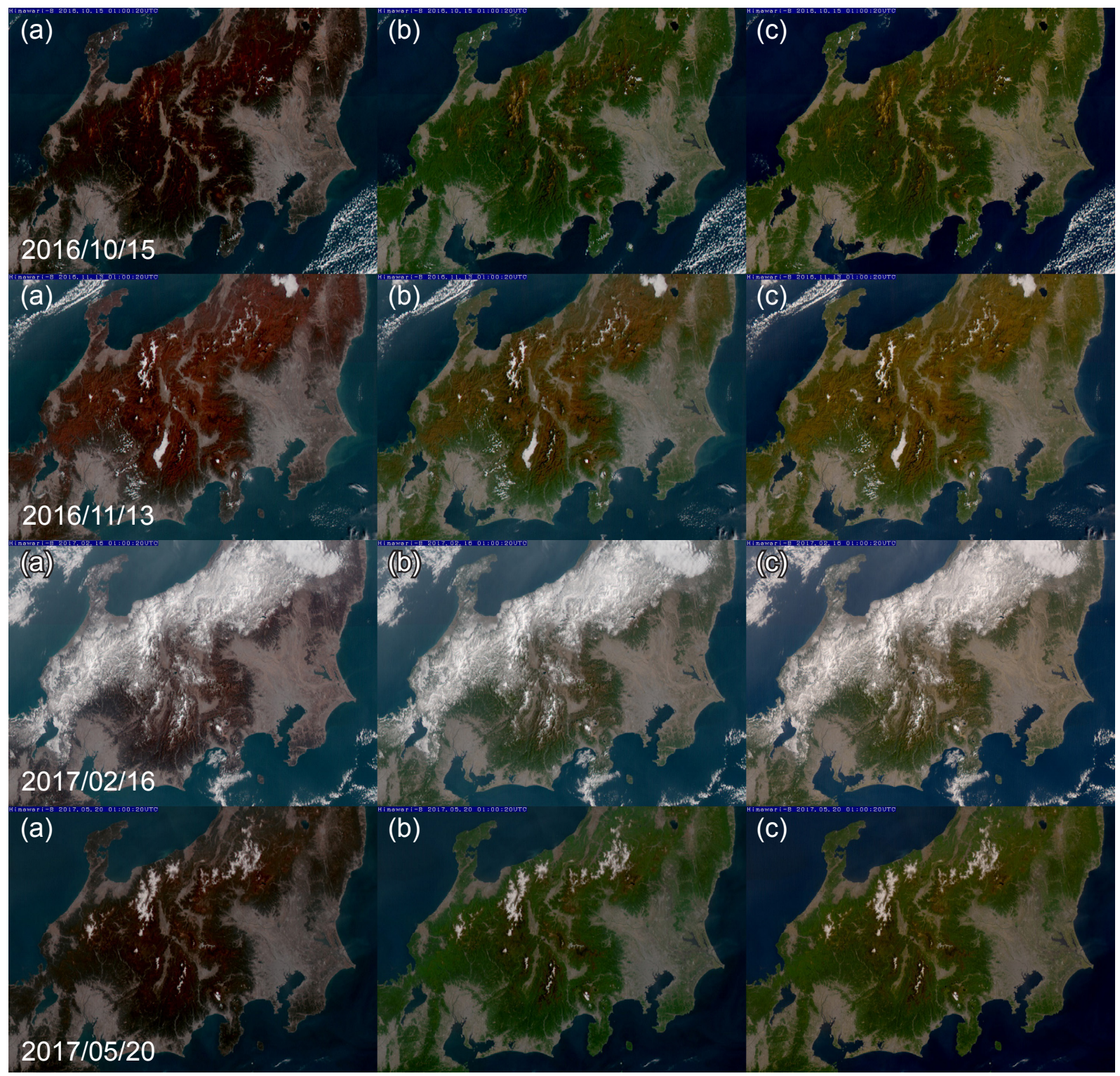

Fig. 25. True color images of central Japan for 15 October 20160100 UTC, 13 November 20160100 UTC, 16 February 20170100 UTC and 20 May 20170100 UTC. (a) Simple composition imagery from AHI native RGB bands, (b) HAC imagery, and (c) TCR imagery. Rayleigh scattering correction and log-linear scaling (see Section 6) are applied to all images.

of SRFs. XYZ values obtained for a surface whose reflectance spectrum has a particular feature such as a local peak outside the coverage of the used SRFs are expected to be less accurate, as the satellite cannot detect signals affected by such feature.

The evaluation technique presented here (see Section 4) can be used to evaluate the accuracy of a color for a given reflectance spectrum even if it is not included in the reflectance spectrum dataset, thereby helping to clarify color precision for a particular reflectance spectrum. Reflectance spectra observed using hyperspectral sensors (e.g., Hyperion) also help highlight color inaccuracies on the actual earth's surface and clarify the limitations of TCR imagery. 


\section{b. Ideal RGB band combination}

In this study, the optimal green band was identified by shifting the SRF via trial and error. However, it remains unclear which combination of RGB bands is ideal for obtaining accurate XYZ values. One possible approach involves changing the shape and width of the SRF and shifting the SRFs of the red and blue bands as well.

\section{c. Colors of floating particles}

As the focus of this study was on the colors of surface materials, the accuracy of colors used to represent floating particles such as dust, smoke, and haze remains unclear. In addition, the dependence on the wavelength of reflection from cloud (ice/water) particles, which can affect color, is not considered based on an assumption of its insignificance within the range of bands used (approx. 0.4 to $0.9 \mu \mathrm{m}$ ). To clarify the detailed accuracy of the colors of floating particles, the dependence on the wavelength of reflection needs to be considered based on the radiative transfer theory.

\section{d. True colors incorporating Rayleigh scattering}

In this study, application of Rayleigh scattering correction to the source satellite imagery enabled color reproduction without the effects of scattering by atmospheric molecules to produce colors as if seen near the surface. The resultant colors therefore differ from those actually seen from space (i.e., with a milky blue feature). The parameters for reproducing colors as seen from space can be similarly derived by replacing the reflectance spectra used in this study with values generated in consideration of Rayleigh scattering.

\section{e. Calibration accuracy}

As inferred by Eq. (12) (or Eq. 21), calibration biases in observation data affect the resultant colors due to changes in the balance of source RGB signals. By way of example, the SRFs of Himawari- 8 and Himawari-9 are very similar, but the reflectance of Himawari-9/B01 $(0.47 \mu \mathrm{m})$ is $4-6 \%$ brighter beyond the SRF difference (Murata et al. 2017). As a result, Himawari-9 TCR imagery without bias correction is slightly bluish compared with that of Himawari-8. As the parameters discussed here were derived without consideration for calibration error, calibration biases need to be removed before all processes.

\section{f. $R G B$ signals for the other standards}

In this study, we assumed that the output devices comply with the sRGB standard, and XYZ values are converted into RGB signals for the standard. The conversion to RGB signals for another standard (e.g., Adobe RGB) is enabled by replacing the conversion matrix (11) with the relevant one for the standard.

As a matter of note, since the printing of this article is based on the CMYK color space, the colors of the imagery shown in this article may be slightly different from those displayed on the sRGB display.

\section{Conclusions}

In this study, we applied a color reproduction approach based on the CIE $1931 \mathrm{XYZ}$ color system to true color imagery rendering for Himawari-8/AHI to reproduce colors seen by the human eye. In this approach, RGB signals (reflectance or scaled radiance) observed by AHI are converted into XYZ tristimulus values, which can express any color independently of standards and devices, and then reconverted into RGB signals for output devices compliant with the SRGB standard. Conversions are performed using $3 \times 3$ matrices generated from the chromaticity coordinates of RGB primary colors and the white point specific to the input and output devices (i.e., satellite and display).

Accurate $\mathrm{XYZ}$ values obtained from satellite observations enable faithful reproduction of colors as seen by the human eye. An objective evaluation technique was developed to check the accuracy of the obtained $\mathrm{XYZ}$ values from AHI observations. The results showed that the combination of AHI native RGB bands was not optimal for obtaining accurate XYZ values. Accordingly, the green band was replaced with a pseudo band with a central wavelength of 0.555 $\mu \mathrm{m}$ as determined using the evaluation technique. Pseudo band observation data were generated using a regression formula with data from existing AHI bands as predictor variables. Differences in color among true color imagery variations were discussed. The differences were consistent with those inferred from the XYZ values using the evaluation technique.

A new type of true color imagery, rendered by this approach in addition to the Rayleigh correction and log-linear scaling provided by CIRA, was named TCR imagery. TCR imagery is beneficial for phenomena that can be identified by color with the human eye, such as dust clouds and seasonal vegetation changes.

\section{Acknowledgment}

The imagery discussed in this study was developed via collaboration between JMA's Meteorological Satellite Center and the NOAA/NESDIS/STAR GOES-R Algorithm Working Group imagery team. The authors are grateful to all involved for their collaborative efforts and for permission to use the related computer 
programs.

\section{Appendix: Derivation of the conversion matrix from RGB to XYZ}

Derivation of the $3 \times 3$ matrix used to convert RGB signals to $\mathrm{XYZ}$ tristimulus values (referred to here as $\mathrm{XYZ}$ values) is described here with reference to Ohta (1997) and Taniguchi and Zhang (2012). RGB signals and $\mathrm{XYZ}$ values can be mutually converted with the matrix and the inverse matrix. The components of the matrix are obtained using the given chromaticity coordinates of the primary colors of light (i.e., red, green, and blue) and the white point, whose symbols are shown in Table 2.

$\mathrm{XYZ}$ values are obtained by adding the respective $\mathrm{XYZ}$ values for individual actions of each RGB primary color. The relation between RGB signals and $\mathrm{XYZ}$ values is described as follows, and the matrix is to be derived:

$$
\left(\begin{array}{c}
X \\
Y \\
Z
\end{array}\right)=\left(\begin{array}{ccc}
X_{R} & X_{G} & X_{B} \\
Y_{R} & Y_{G} & Y_{B} \\
Z_{R} & Z_{G} & Z_{B}
\end{array}\right)\left(\begin{array}{l}
R \\
G \\
B
\end{array}\right)
$$

where $\mathrm{R}, \mathrm{G}$, and $\mathrm{B}$ are RGB signals varying between 0 and 1. $\left(X_{R}, Y_{R}, Z_{R}\right)$ are the $\mathrm{XYZ}$ values of $\mathrm{R}$ alone, whereas $\left(X_{G}, Y_{G}, Z_{G}\right)$ and $\left(X_{B}, Y_{B}, Z_{B}\right)$ are those of $\mathrm{G}$ and $\mathrm{B}$.

Summations of the XYZ values for each primary color taken as $S_{R}, S_{G}$, and $S_{B}$ are written as follows:

$$
\begin{aligned}
& S_{R}=X_{R}+Y_{R}+Z_{R}, \\
& S_{G}=X_{G}+Y_{G}+Z_{G}, \\
& S_{B}=X_{B}+Y_{B}+Z_{B} .
\end{aligned}
$$

The relation between the given chromaticity coordinates $\left(x_{R}, y_{R}, z_{R}\right)$ and $\mathrm{XYZ}$ values $\left(X_{R}, Y_{R}, Z_{R}\right)$ is expressed using $S_{R}$ for the primary color R as follows:

$$
\begin{aligned}
& x_{R}=\frac{X_{R}}{X_{R}+Y_{R}+Z_{R}}=\frac{X_{R}}{S_{R}}, \\
& y_{R}=\frac{Y_{R}}{X_{R}+Y_{R}+Z_{R}}=\frac{Y_{R}}{S_{R}}, \\
& z_{R}=\frac{Z_{R}}{X_{R}+Y_{R}+Z_{R}}=\frac{Z_{R}}{S_{R}} .
\end{aligned}
$$

The following are obtained by rearranging the above and can be similarly applied for $\mathrm{G}$ and $\mathrm{B}$ :

$$
\begin{aligned}
& \left(\begin{array}{c}
X_{R} \\
Y_{R} \\
Z_{R}
\end{array}\right)=S_{R}\left(\begin{array}{l}
x_{R} \\
y_{R} \\
z_{R}
\end{array}\right), \\
& \left(\begin{array}{c}
X_{G} \\
Y_{G} \\
Z_{G}
\end{array}\right)=S_{G}\left(\begin{array}{l}
x_{G} \\
y_{G} \\
z_{G}
\end{array}\right), \\
& \left(\begin{array}{c}
X_{B} \\
Y_{B} \\
Z_{B}
\end{array}\right)=S_{B}\left(\begin{array}{l}
x_{B} \\
y_{B} \\
z_{B}
\end{array}\right) .
\end{aligned}
$$

The matrix is obtained by assembling the above as follows:

$$
\left(\begin{array}{ccc}
X_{R} & X_{G} & X_{B} \\
Y_{R} & Y_{G} & Y_{B} \\
Z_{R} & Z_{G} & Z_{B}
\end{array}\right)=\left(\begin{array}{lll}
x_{R} & x_{G} & x_{B} \\
y_{R} & y_{G} & y_{G} \\
z_{R} & z_{G} & z_{B}
\end{array}\right)\left(\begin{array}{ccc}
S_{R} & 0 & 0 \\
0 & S_{G} & 0 \\
0 & 0 & S_{B}
\end{array}\right) .
$$

Equation (A1) can then be rewritten as follows:

$$
\left(\begin{array}{l}
X \\
Y \\
Z
\end{array}\right)=\left(\begin{array}{lll}
x_{R} & x_{G} & x_{B} \\
y_{R} & y_{G} & y_{G} \\
z_{R} & z_{G} & z_{B}
\end{array}\right)\left(\begin{array}{ccc}
S_{R} & 0 & 0 \\
0 & S_{G} & 0 \\
0 & 0 & S_{B}
\end{array}\right)\left(\begin{array}{l}
R \\
G \\
B
\end{array}\right) .
$$

To obtain $\left(S_{R}, S_{R}, S_{R}\right)$, XYZ values for the white point expressed as $\left(X_{W}, Y_{W}, Z_{W}\right)$ and their summation expressed as $S_{W}$ are written as follows:

$$
S_{W}=X_{W}+Y_{W}+Z_{W} .
$$

The relation between the given chromaticity coordinates of the white point $\left(x_{W}, y_{W}, z_{W}\right)$ and $\left(X_{W}, Y_{W}, Z_{W}\right)$ can then be expressed as in Eq. (A3) as follows:

$$
\begin{gathered}
x_{W}=\frac{X_{W}}{X_{W}+Y_{W}+Z_{W}}=\frac{X_{W}}{S_{W}}, \\
y_{W}=\frac{Y_{W}}{X_{W}+Y_{W}+Z_{W}}=\frac{Y_{W}}{S_{W}}, \\
z_{W}=\frac{Z_{W}}{X_{W}+Y_{W}+Z_{W}}=\frac{Z_{W}}{S_{W}} .
\end{gathered}
$$

The tristimulus value $\mathrm{Y}$ representing luminance is normalized to $Y_{W}=1$ and substituted into the expression of $y_{W}$ in Eq. (A8). $S_{W}$ is derived as follows:

$$
S_{W}=\frac{1}{y_{W}} .
$$

From Eqs. (A8) and (A9), $\left(X_{W}, Y_{W}, Z_{W}\right)$ are obtained with the given chromaticity coordinates $\left(x_{W}, y_{W}, z_{W}\right)$ as follows: 


$$
\left(\begin{array}{c}
X_{W} \\
Y_{W} \\
Z_{W}
\end{array}\right)=S_{W}\left(\begin{array}{c}
x_{W} \\
y_{W} \\
z_{W}
\end{array}\right)=\left(\begin{array}{c}
\frac{x_{W}}{y_{W}} \\
1 \\
\frac{z_{W}}{y_{W}}
\end{array}\right),
$$

$\left(X_{W}, Y_{W}, Z_{W}\right)$ are obtained when $R=G=B=1$, and the following is obtained via substitution into Eq. (A6):

$$
\begin{aligned}
\left(\begin{array}{l}
X_{W} \\
Y_{W} \\
Z_{W}
\end{array}\right) & =\left(\begin{array}{lll}
x_{R} & x_{G} & x_{B} \\
y_{R} & y_{G} & y_{B} \\
z_{R} & z_{G} & z_{B}
\end{array}\right)\left(\begin{array}{ccc}
S_{R} & 0 & 0 \\
0 & S_{G} & 0 \\
0 & 0 & S_{B}
\end{array}\right)\left(\begin{array}{l}
1 \\
1 \\
1
\end{array}\right) \\
& =\left(\begin{array}{lll}
x_{R} & x_{G} & x_{B} \\
y_{R} & y_{G} & y_{B} \\
z_{R} & z_{G} & z_{B}
\end{array}\right)\left(\begin{array}{l}
S_{R} \\
S_{G} \\
S_{B}
\end{array}\right) .
\end{aligned}
$$

From Eqs. (A10) and (A11), the unknown parameters $\left(S_{R}, S_{G}, S_{B}\right)$ are obtained using the given chromaticity coordinates as follows:

$$
\begin{aligned}
\left(\begin{array}{l}
S_{R} \\
S_{G} \\
S_{B}
\end{array}\right) & =\left(\begin{array}{lll}
x_{R} & x_{G} & x_{B} \\
y_{R} & y_{G} & y_{B} \\
z_{R} & z_{G} & z_{B}
\end{array}\right)^{-1}\left(\begin{array}{c}
X_{W} \\
Y_{W} \\
Z_{W}
\end{array}\right) \\
& =\left(\begin{array}{lll}
x_{R} & x_{G} & x_{B} \\
y_{R} & y_{G} & y_{B} \\
z_{R} & z_{G} & z_{B}
\end{array}\right)^{-1}\left(\begin{array}{c}
\frac{x_{W}}{y_{W}} \\
1 \\
\frac{z_{W}}{y_{W}}
\end{array}\right) .
\end{aligned}
$$

Substituting the obtained values of $\left(S_{R}, S_{G}, S_{B}\right)$ into Eq. (A5) gives the desired $3 \times 3$ conversion matrix in Eq. (A1).

\section{References}

American Society for Testing \& Materials, 2000: 2000 ASTM standard extraterrestrial solar spectrum reference E-490-00. Grid modernization. NREL Transforming Energy. [Available at http://rredc.nrel.gov/ solar/spectra/am0/.]

Bessho, K., K. Date, M. Hayashi, A. Ikeda, T. Imai, H. Inoue, Y. Kumagai, T. Miyakawa, H. Murata, T. Ohno, A. Okuyama, R. Oyama, Y. Sasaki, Y. Shimazu, K. Shimoji, Y. Sumida, M. Suzuki, H. Taniguchi, H. Tsuchiyama, D. Uesawa, H. Yokota, and R. Yoshida, 2016: An introduction to Himawari-8/9—Japan's newgeneration geostationary meteorological satellites. $J$. Meteor. Soc. Japan, 94, 151-183.

CIE, 1932: Commission Internationale de l'éclairage Proceedings, 1931. Huitième session. Cambridge University Press, 19-29.

Clark, R. N., G. A. Swayze, R. A. Wise, K. E. Livo, T. M.
Hoefen, R. F. Kokaly, and S. J. Sutley, 2007: USGS Digital Spectral Library splib06a'. U.S. Geological Survey Data Series, 231, [Available at http://speclab. cr.usgs.gov/spectral.lib06/ds231.]

Griffith, P. C., and K.-H. Yang, 2016: Significant increase in geostationary imaging temporal resolution for GEOKOMPSAT-2A AMI. The seventh Asia/Oceania Meteorological Satellite Users' Conference (AOMSUC-7), Songdo, Korea, October, 2016, 10 pp.

Giorgianni, E. J., and T. E. Madden, 2008: Digital Color Management: Encoding Solutions. John Wiley \& Sons, $432 \mathrm{pp}$.

International Electrotechnical Commission, 1999: Multimedia systems and equipment - Colour measurement and management-Part 2-1: Colour management - Default RGB colour space - sRGB. IEC, 61966-2-1, 51 pp.

ISO/CIE, 2009: Colorimetry—Part 5: CIE $1976 \mathrm{~L}^{*} \mathrm{u}^{*} \mathrm{v}^{*}$ colour space and $\mathrm{u}^{\prime}, \mathrm{v}^{\prime}$ uniform chromaticity scale diagram. ISO 11664-5:2009(en). [Available at https:// www.iso.org/obp/ui/\#iso:std:iso:11664:-5:ed-1:v1:en.]

Japan Meteorological Agency, 2017: First images from Himawari-9. News Release, 24 January, 2017. [Available at http://www.jma.go.jp/jma/jma-eng/satellite/ news/himawari89/20170124_himawari9_first_images. html.]

Miller, S. D., C. C. Schmidt, T. J. Schmit, and D. W. Hillger, 2012: A case for natural colour imagery from geostationary satellites, and an approximation for the GOES-R ABI. Int. J. Remote Sens., 33, 3999-4028.

Miller, S. D., T. L. Schmit, C. J. Seaman, D. T. Lindsey, M. M. Gunshor, R. A. Kohrs, Y. Sumida, and D. Hillger, 2016: A sight for sore eyes-The return of true color to geostationary satellites. Bull. Amer. Meteor. Soc., 97, 1803-1816.

Mohr, T., 2014: Preparing the use of new generation geostationary meteorological satellites. WMO Bull., 63, $42-44$.

Murata, H., T. Tabata, A. Okuyama, and M. Takahashi, 2017: Inter-comparison of Himawari-8/-9 AHI using a GEOGEO approach. GSICS Quarterly, 11, doi:10.7289/ V5BK19F0.

NASA, 2015: Aqua Product Descriptions: MYD09. MODAPS Services. [Available at https://modaps. modaps.eosdis.nasa.gov/services/about/products/c6nrt/MYD09.html.]

Ohta, N., 1997: Introduction to Color Reproduction Technology. Corona Publishing Co., Ltd. 266 pp (in Japanese).

Schmit, T. J., M. M. Gunshor, W. P. Menzel, J. J. Gurka, J. $\mathrm{Li}$, and A. S. Bachmeier, 2005: Introducing the nextgeneration Advanced Baseline Imager on GOES-R. Bull. Amer. Meteor. Soc., 86, 1079-1096.

Schmit, T. J., J. Li, J. J. Gurka, M. D. Goldberg, K. J. Schrab, J. Li, and W. F. Feltz, 2008: The GOES-R Advanced Baseline Imager and the continuation of current sounder products. J. Appl. Meteor. Climatol., 47, 2696-2711. 
Schmit, T. J., P. Griffith, M. M. Gunshor, J. M. Daniels, S. J. Goodman, and W. J. Lebair, 2017: A closer look at the ABI on the GOES-R series. Bull. Amer. Meteor. Soc., 98, 681-698.

Stöckli, R., E. Vermote, N. Saleous, R. Simmon, and D. Herring, 2007: The Blue Marble Next Generation-A true color earth dataset including seasonal dynamics from MODIS. NASA Earth Observatory, $12 \mathrm{pp}$.

Taniguchi, K., and X. Zhang, 2012: Digital Color Engineer- ing. Kyoritsu Shuppan, 240 pp (in Japanese).

Vidot, J., and É. Borbás, 2014: Land surface VIS/NIR BRDF atlas for RTTOV-11: Model and validation against SEVIRI land SAF albedo product. Quart. J. Roy. Meteor. Soc., 140, 2186-2196.

Yang, J., Z. Zhang, C. Wei, F. Lu, and Q. Guo, 2017: Introducing the new generation of Chinese geostationary weather satellites-Fengyun-4. Bull. Amer. Meteor. Soc., 98, 1637-1658. 